\title{
OPEN Comprehensive transcriptomic analysis of COVID-19 blood, lung, and airway
}

\author{
Andrea R. Daamen ${ }^{1,2 \bowtie}$, Prathyusha Bachali ${ }^{1,2}$, Katherine A. Owen ${ }^{1,2}$, \\ Kathryn M. Kingsmore ${ }^{1,2}$, Erika L. Hubbard ${ }^{1,2}$, Adam C. Labonte ${ }^{1}$, Robert Robl ${ }^{1}$, \\ Sneha Shrotri ${ }^{1}$, Amrie C. Grammer ${ }^{1}$ \& Peter E. Lipsky ${ }^{1 凶}$
}

SARS-CoV2 is a previously uncharacterized coronavirus and causative agent of the COVID-19 pandemic. The host response to SARS-CoV2 has not yet been fully delineated, hampering a precise approach to therapy. To address this, we carried out a comprehensive analysis of gene expression data from the blood, lung, and airway of COVID-19 patients. Our results indicate that COVID-19 pathogenesis is driven by populations of myeloid-lineage cells with highly inflammatory but distinct transcriptional signatures in each compartment. The relative absence of cytotoxic cells in the lung suggests a model in which delayed clearance of the virus may permit exaggerated myeloid cell activation that contributes to disease pathogenesis by the production of inflammatory mediators. The gene expression profiles also identify potential therapeutic targets that could be modified with available drugs. The data suggest that transcriptomic profiling can provide an understanding of the pathogenesis of COVID-19 in individual patients.

Coronaviruses $(\mathrm{CoV})$ are a group of enveloped, single, positive-stranded RNA viruses causing mild to severe respiratory illnesses in humans ${ }^{1-3}$. In the past two decades, three worldwide outbreaks have originated from CoVs capable of infecting the lower respiratory tract, resulting in heightened pathogenicity and high mortality rates. We are currently in the midst of a pandemic stemming from a third $\mathrm{CoV}$ strain, severe acute respiratory syndrome coronavirus 2 (SARS-CoV2), the causative agent of coronavirus disease 2019 (COVID-19). In the majority of cases, patients exhibit mild symptoms, whereas in more severe cases, patients may develop severe lung injury and death from respiratory failure ${ }^{4,5}$.

At this time, there is still incomplete information available regarding the host response to SARS-CoV2 infection and the perturbations resulting in a severe outcome. Despite this, clues can be derived from our knowledge of the immune response to infection by other respiratory viruses, including other CoVs. After infection, viruses are typically detected by pattern recognition receptors (PRRs) such as the inflammasome sensor NLRP3, which signal the release of interferons (IFNs) and inflammatory cytokines including the IL-1 family, IL-6, and TNF, that activate a local and systemic response to infection ${ }^{6,7}$. This involves the recruitment, activation, and differentiation of innate and adaptive immune cells, including neutrophils, inflammatory myeloid cells, CD8 T cells, and natural killer (NK) cells ${ }^{8}$. Resolution of infection is largely dependent on the cytotoxic activity of CD8 T cells and NK cells, which enable clearance of virus-infected cells ${ }^{8}$. Failure to clear virus-infected cells may facilitate a hyper-inflammatory state termed Macrophage (MФ) activation syndrome (MAS) or "cytokine storm", and ultimately damage to the infected lung 9,10 .

The recent emergence of SARS-CoV2 and the relative lack of comprehensive knowledge regarding the progression of COVID-19 disease has constrained our ability to develop effective treatments for infected patients. One means to obtain a more complete understanding of the host response to SARS-CoV2 is to examine gene expression in relevant tissues. A limited number of gene expression profiles are available from patients with COVID-19 and have yielded some insights into the pathogenic processes triggered by infection with SARS$\mathrm{CoV} 2^{11-13}$. However, because of the small number of samples and limited analysis, a full picture of the biological state of SARS-CoV2-affected tissues has not emerged. To address this, we have assessed available SARS-CoV2 gene expression datasets from blood, lung, and airway using a number of orthogonal bioinformatic tools to provide a more complete view of the nature of the COVID-19 inflammatory response and the potential points of therapeutic intervention.

\footnotetext{
${ }^{1}$ AMPEL BioSolutions LLC, Charlottesville, VA 22902, USA. ${ }^{2}$ These authors contributed equally: Andrea R. Daamen, Prathyusha Bachali, Katherine A. Owen, Kathryn M. Kingsmore and Erika L. Hubbard. ${ }^{\varpi}$ email: andrea.daamen@ ampelbiosolutions.com; peterlipsky@comcast.net
} 


\section{Results}

Gene expression analysis of blood, lung, and airway of COVID-19 patients. To characterize the pathologic response to SARS-CoV2 infection, we analyzed transcriptomic data from peripheral blood mononuclear cells (PBMCs) and postmortem lung tissue of COVID-19 patients and healthy controls as well as bronchoalveolar lavage (BAL) fluid of COVID-19 patients (CRA002390, GSE147507, Supplementary Fig. 1) ${ }^{11,12}$. We first determined changes in gene expression in the blood (PBMC-CTL vs PBMC-CoV2) and lung (Lung-CTL vs Lung-CoV2). Because no control BAL fluids were associated with the BAL-CoV2 samples, we compared BAL-CoV2 to PBMC-CoV2 from the same dataset to avoid effects related to batch and methodology. Overall, we found 4245 differentially expressed genes (DEGs) in the blood (2166 up and 2079 down), 2220 DEGs in the lung (684 up and 1536 down), and 8952 DEGs in the airway (BAL) (4052 up and 4900 down) (Supplementary Table 2).

Conserved and differential enrichment of inflammatory cells and pathways in COVID-19 patients. To interrogate pathologic pathways in the 3 compartments, we carried out Gene Set Variation Analysis (GSVA) utilizing a number of informative gene modules ${ }^{15,16}$ (Fig. 1, Supplementary Table 3). Numerous innate immune response pathways were increased in all 3 compartments, whereas adaptive immune signatures tended to be decreased in blood and airway, but not lung, a pattern that is consistent with earlier studies ${ }^{11}$, ${ }_{12,17}$. Although heterogeneity was observed in all compartments, closer examination revealed consistent perturbations. In the blood, the classical and lectin-induced complement pathways, as well as the NLRP3 inflammasome, plasma cells (PCs), and monocytes (Mo) were significantly enriched in COVID-19 patients, whereas cytotoxic cells and neutrophils were decreased. In the lung, the NLRP3 inflammasome, Mo and myeloid cells were enriched in COVID-19 patients. In addition, although the general granulocyte signature was not significantly increased, a specific low-density granulocyte (LDG) signature ${ }^{18}$ and gene sets of inflammatory and suppressive neutrophils previously derived from COVID-19 blood were enriched in the lung ${ }^{19,20}$. In the airway, the classical and alternative complement pathways were enriched and T cells and cytotoxic cells were decreased.

Although there is conflicting data on the presence of an IFN gene signature (IGS) and whether SARS-CoV2 infection induces a robust IFN response ${ }^{12,13,21}$, we observed increased expression of Type I IFN genes (IFNA4, IFNA6, IFNA10) and significant enrichment of the common Type I and Type II IGS, including enrichment of IFNA2, IFNB1 and IFNG gene signatures specifically in the lung tissue (Fig. 2a,b). Furthermore, we detected increased expression of genes found to be important for the anti-viral innate immune response (IFIH1, DDX58, EIF2AK2, OAS2) and decreased expression of negative regulators of this response (IRF2BP1, SKIV2L) in both the lung and airway compartments (Fig. 2c) ${ }^{22}$. Interestingly, we found expression of MAVS, a signaling adaptor for RNA virus sensors, was decreased in the airway, which is consistent with the reported effect of SARS-CoV2 and may reflect a mechanism of viral immune evasion ${ }^{23,24}$.

Increased expression of inflammatory mediators in the lungs of COVID-19 patients. To examine the nature of the inflammatory response in the tissue compartments in greater detail, we examined specific DEGs of interest (Fig. 3a,b). In the blood, we noted increased expression of the inflammatory chemokine CXCL10, which is an IFNG response gene and involved in the activation and chemotaxis of peripheral immune cells $^{25}$, the chemokine receptor CCR2, which has been shown to be critical for immune cell recruitment in response to respiratory viral infection ${ }^{26}$, as well as the inflammatory IL-1 family member, IL18. Expression of a number of chemokines, including ligands for CCR2, were significantly increased in both the lung tissue and airway of COVID-19 patients, including CCL2, CCL3L1, CCL7, CCL8, and CXCL10. We also observed elevated pro-inflammatory IL-1 family members, ILIA and $I L 1 B$, in these 2 compartments. Furthermore, lung tissue exhibited enrichment of the IL-1 cytokine gene signature, whereas the airway exhibited additional expression of IL18, IL33, IL36B, and IL36G.

Non-hematopoietic cells in the BAL fluid may be indicative of viral-induced damage. To determine whether viral infection resulted in modification of resident tissue populations, we employed GSVA with various non-hematopoietic cell gene signatures (Fig. 3c). We found that signatures of various lung tissue cells but not endothelial cells were enriched in the airway, but not the lung of COVID-19 subjects. Additionally, we also detected increased expression of the viral entry genes ACE2 and TMPRSS2, which are typically expressed on lung epithelium ${ }^{27}$ (Fig. 3d).

Protein-protein interactions identify myeloid subsets in COVID-19 patients. We next sought to utilize an unbiased, protein-protein interaction (PPI)-based clustering approach to assess the inflammatory cell types within each tissue compartment. PPI networks predicted from DEGs were simplified into metastructures defined by the number of genes in each cluster, the number of significant intra-cluster connections, and the number of associations connecting members of different clusters to each other (Fig. 4a-c, Supplementary Table 4). Overall, upregulated PPI networks identified numerous specific cell types and functions. In the blood, cluster 8 was dominated by a Mo population expressing C2, C5, CXCL10, CCR2, and multiple IFN-stimulated genes, whereas cluster 3 contained hallmarks of alternatively activated (M2) MФs and/or myeloid-derived suppressor cells (MDSCs), including CD33, CD36, CD93, and ITGAM (Fig. 4a). Smaller immune clusters were indicative of functions, including inflammasome activation, damage-associated molecular pattern (DAMP) activity, the classical complement cascade and the response to Type II IFNs. Myeloid heterogeneity in the blood was also reflected by the presence of multiple metabolic pathways, such as enhanced oxidative phosphorylation (OXPHOS) in cluster 1 linked to M2-like MФs in cluster 3 (mean interaction score of 0.875), and glycolysis in clusters 7 and 13 connected to activated Mo in cluster 8 (interaction scores of 0.86 and 0.82 , respectively). Con- 


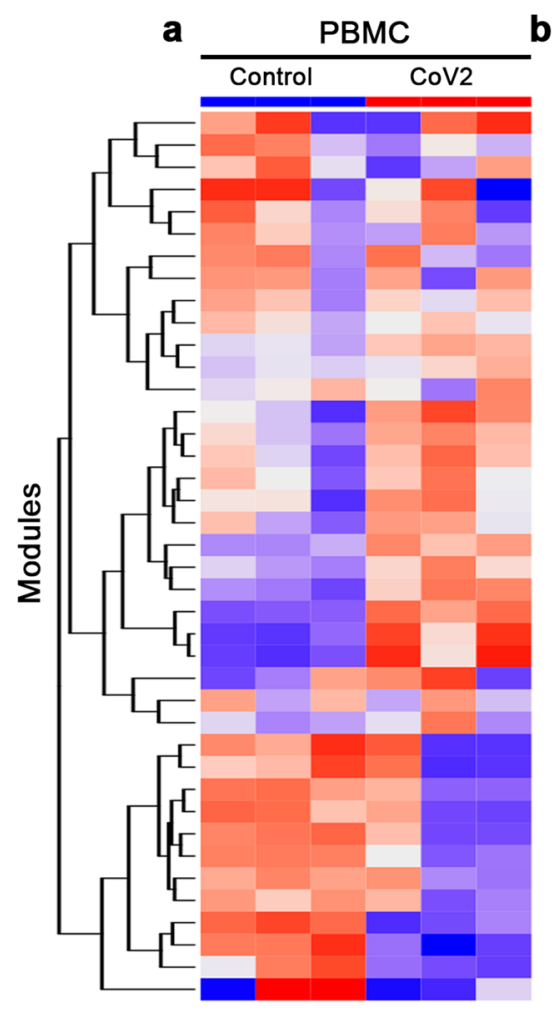

b Lung c

C BAL

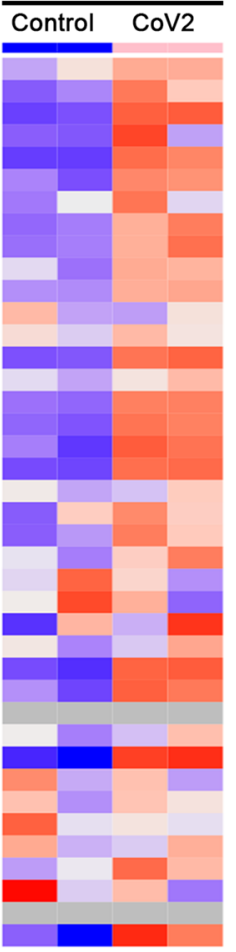

PBMC-CoV2 BAL-CoV2

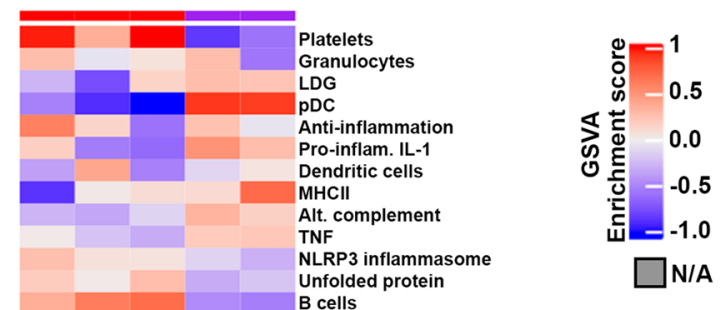

d
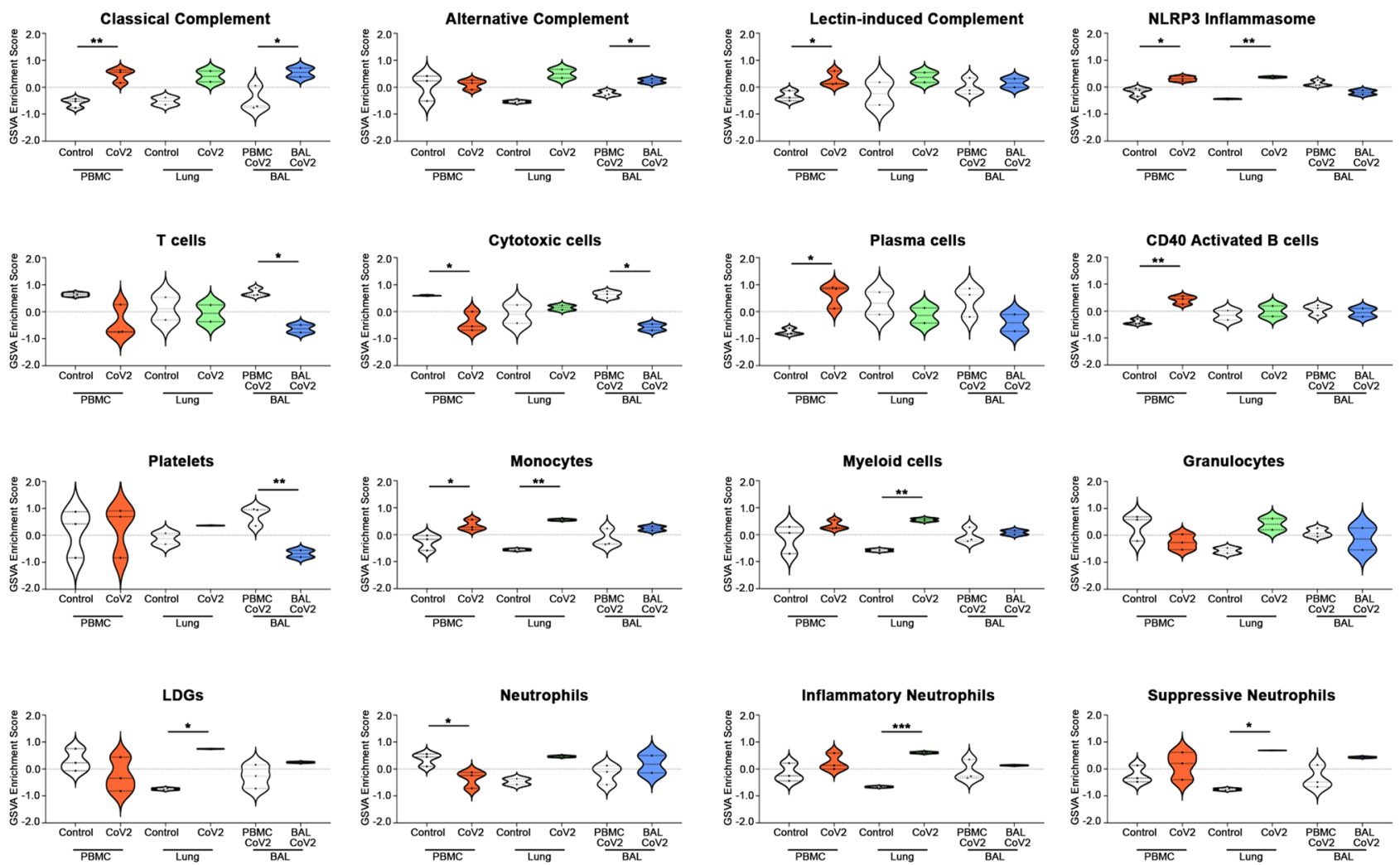

Figure 1. Conserved and differential enrichment of immune cells and pathways in blood, lung, and airway of SARS-CoV2-infected patients. (a-d) Individual sample gene expression from the blood (a), lung (b), and airway (c) was analyzed by GSVA for enrichment of immune cell and inflammatory pathways. The corresponding heatmap was generated using the R Bioconductor package complexHeatmap (v2.5.6) ${ }^{14}$. Select enrichment scores are shown as violin plots in (d) generated using GraphPad Prism v8.4.2 (www.graphpad.com). ${ }^{*} p<0.05$, ${ }^{* *} p<0.01$. 

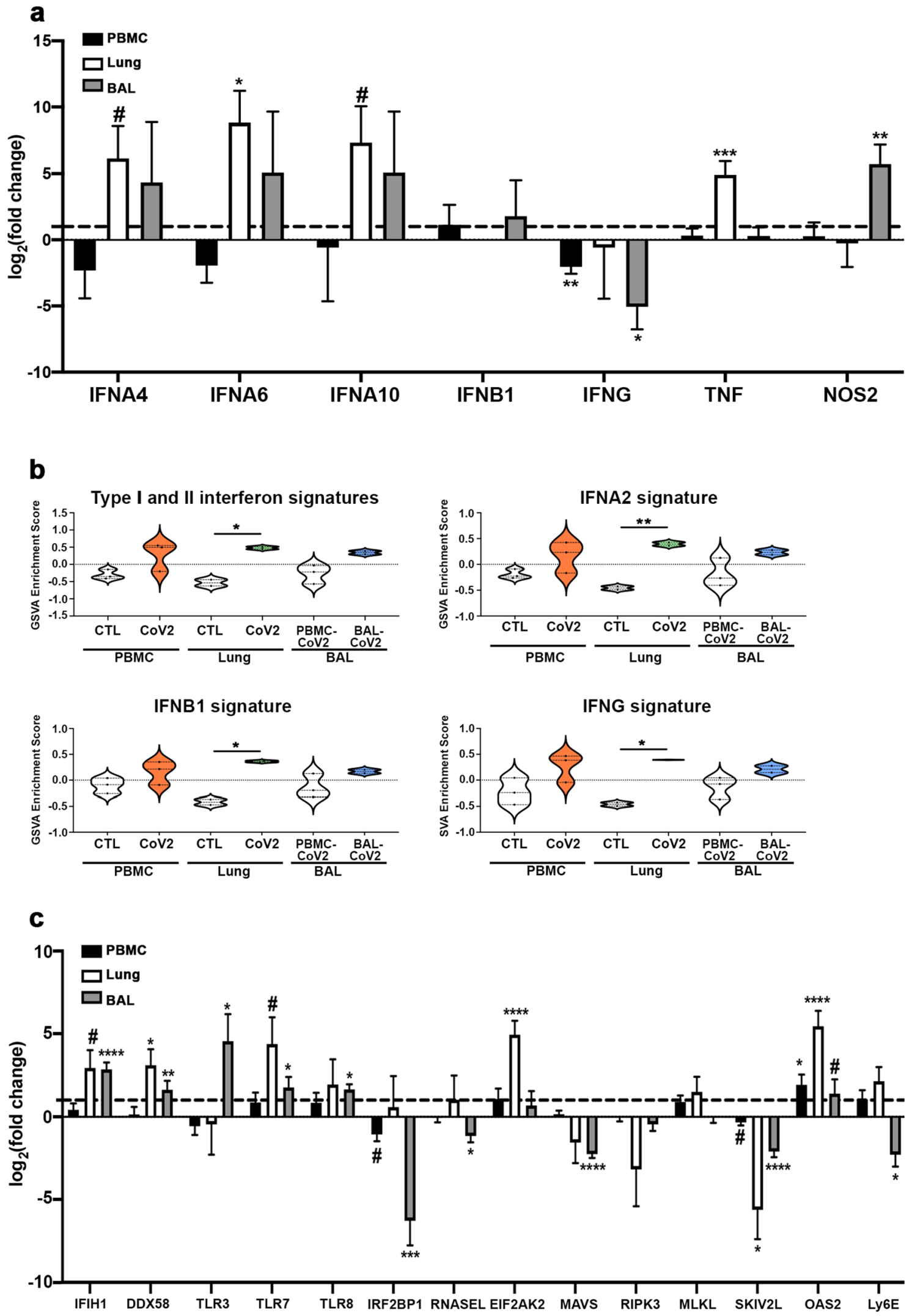

Figure 2. Elevated IFN expression in the lung tissue of COVID-19 patients. (a) Normalized $\log _{2}$ fold change RNA-seq expression values for IFN-associated genes from blood, lung, and airway of individual COVID-19 patients. The dotted line represents the expression of each gene in healthy individuals (for blood and lung) or PBMCs from COVID-19 patients (airway). (b) Individual sample gene expression from the blood, lung, and airway was analyzed by GSVA for enrichment of IFN-related gene signatures. (c) Normalized $\log _{2}$ fold change RNA-seq expression values for anti-viral genes as in (a). Generated using GraphPad Prism v8.4.2 (www.graphpad.com). $\# p<0.2, \# \# p<0.1,{ }^{*} p<0.05,{ }^{*} p<0.01$, ${ }^{* * *} p<0.001,{ }^{* * * *} p<0.0001$. 
a

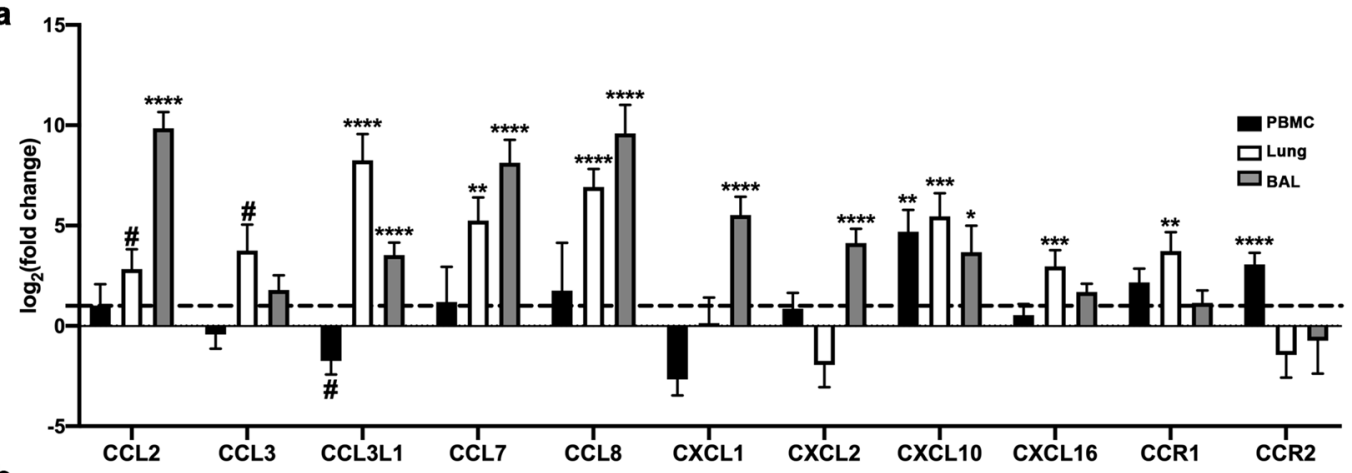

b

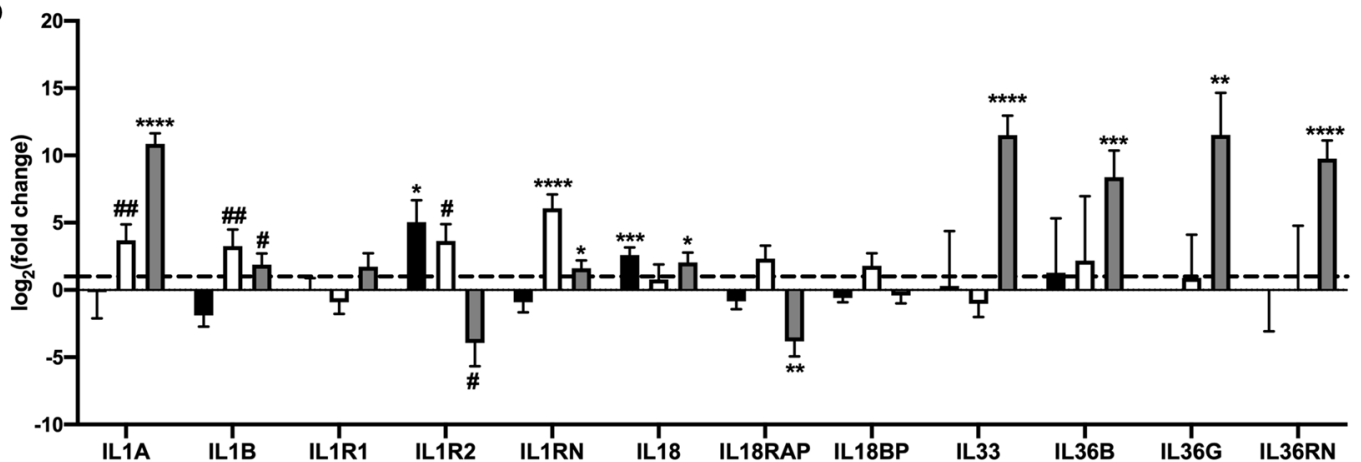

C
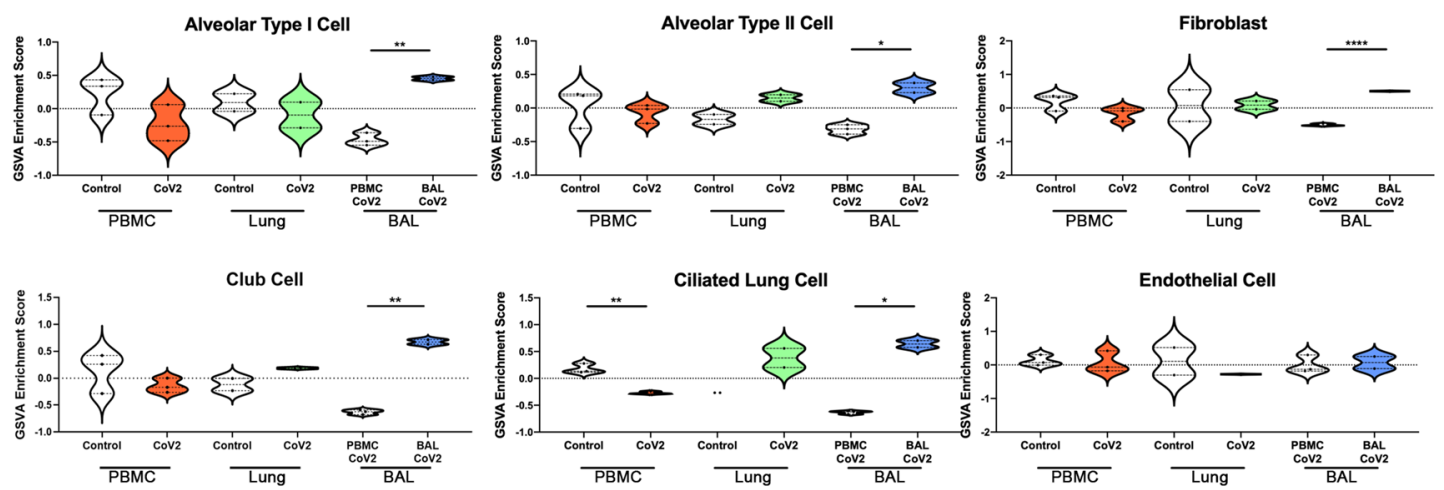

d

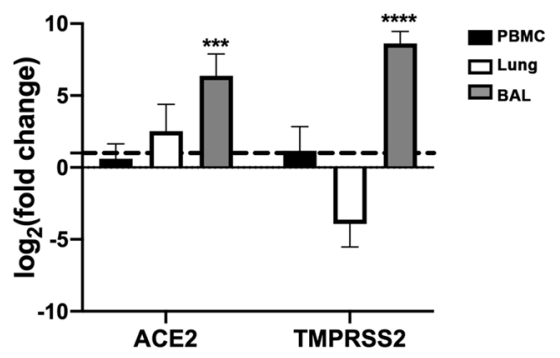

Figure 3. Viral entry gene expression correlates with enhanced expression of inflammatory mediators in SARS-CoV2-infected lungs. (a,b) Normalized $\log _{2}$ fold change RNA-seq expression values for chemokines and chemokine receptors (a) and IL-1 family members (b) from blood, lung, and airway of COVID-19 patients as in Fig. 2a. (c) Individual sample gene expression from the blood, lung, and airway was analyzed by GSVA for enrichment of various lung tissue cell categories. (d) Normalized $\log _{2}$ fold change RNA-seq expression values for viral entry genes as in (a,b). Generated using GraphPad Prism v8.4.2 (www.graphpad.com). \#p<0.2, \#\#p<0.1, ${ }^{*} p<0.05,{ }^{* *} p<0.01,{ }^{* * *} p<0.001,{ }^{* * * *} p<0.0001$.

sistent with our GSVA results, blood exhibited profoundly decreased T cells determined by the downregulation of T cell activation markers CD28, LCK and ITK (Supplementary Fig. 1a).

In addition to the various Mo/myeloid populations, lung tissue was infiltrated by LDGs, granulocytes, $\mathrm{T}$ cells, and B cells. Metabolic function in the lung was varied, with Mo-enriched clusters (1 and 7) linked to glycolysis in cluster 18 (interaction scores of 0.74 and 0.87 , respectively) potentially reflecting cellular activation, whereas OXPHOS was predominantly downregulated along with other nuclear processes (transcription and mRNA 
a

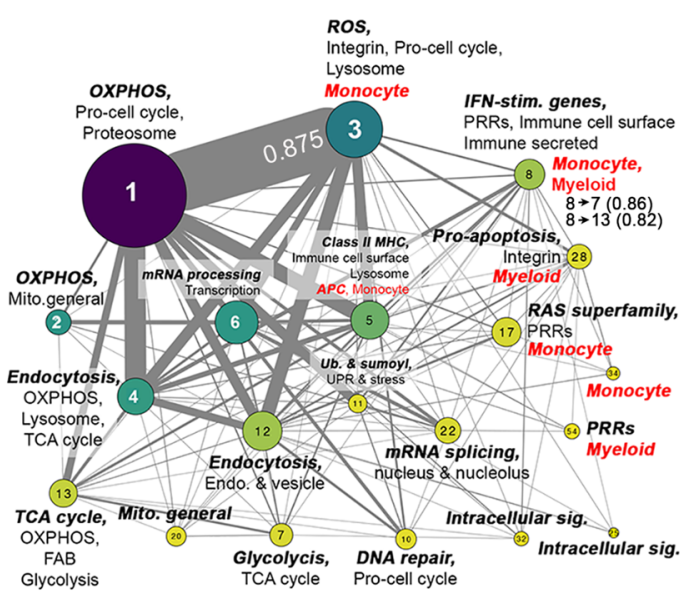

b

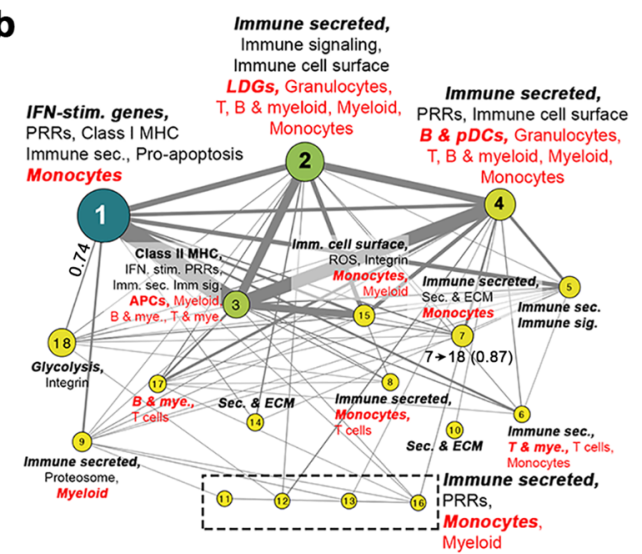

C

BAL

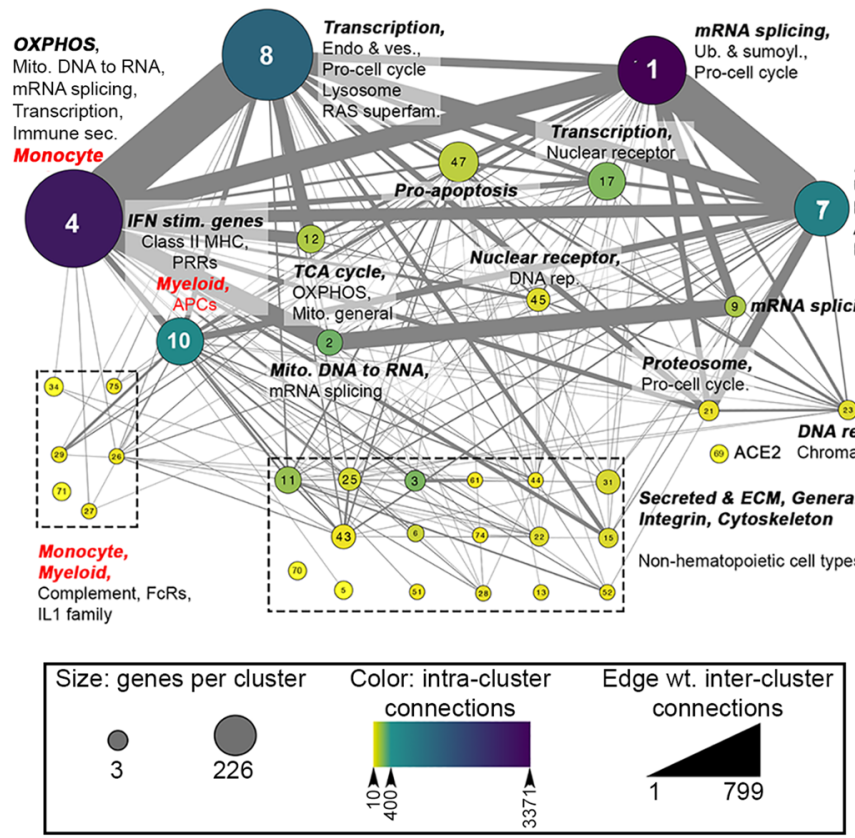

d

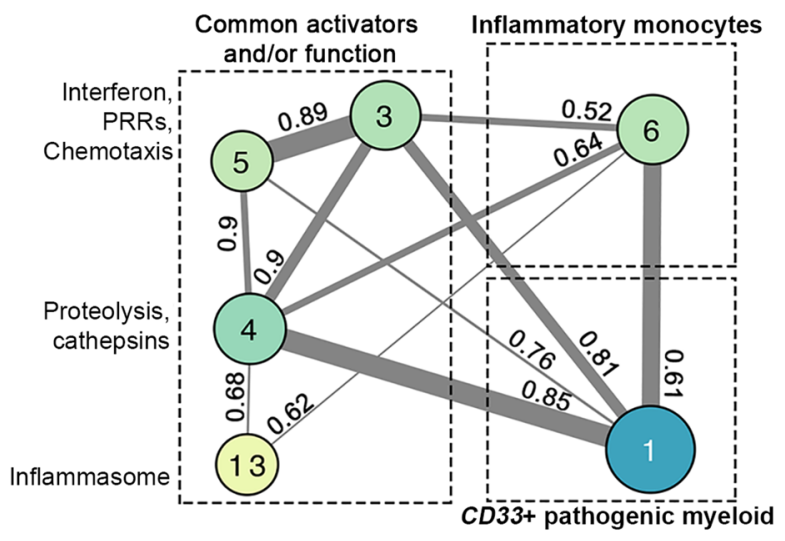

LUNG-Myeloid

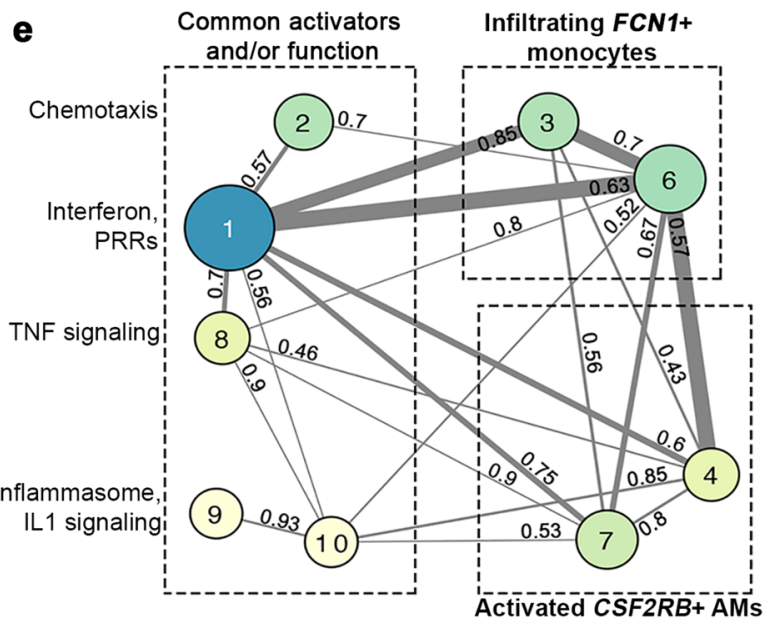

BAL-Myeloid

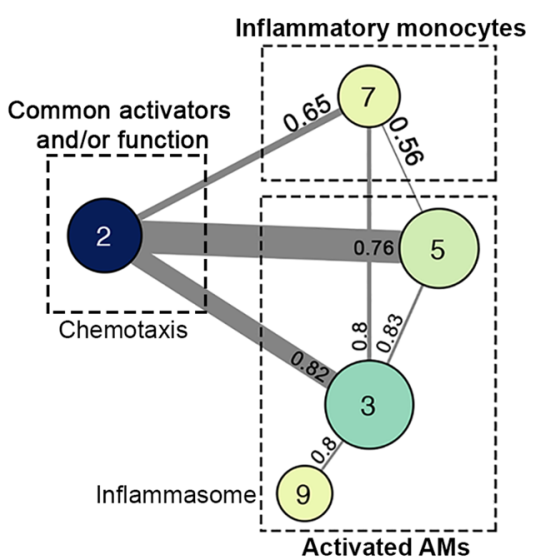


4Figure 4. PPI analysis identifies different myeloid cell subsets and metabolic pathways in blood, lung, and airway of COVID-19 patients. DE upregulated genes from blood (a), lung (b) and airway (c) were used to create PPI metaclusters using Cytoscape (v3.6.1) and the clusterMaker2 (v1.2.1) plugin ${ }^{28}$. Size indicates the number of genes per cluster, color indicates the number of intra-cluster connections and edge weight indicates the number of inter-cluster connections. Enrichment for biological function and immune cell type was determined by BIG-C and I-Scope, respectively. Small clusters ( 14 genes) with similar function are grouped in dotted-line boxes. Clusters enriched in $\mathrm{Mo} /$ myeloid genes were combined by decreasing cluster stringency to create a new set of myeloid-derived metastructures from the blood (d), lung (e) and airway (f). Interaction scores showing the strength of interaction between clusters are indicated (0.4-0.6, medium interaction; $0.61-0.8$, strong interaction; 0.81-0.99, very strong interaction).

processing) (Fig. 4b, Supplementary Fig. 1b). The airway was enriched in inflammatory Mo, mitochondrial function and transcription. Multifunctional cluster 4 was dominated by numerous chemokine and cytokine receptor-ligand pairs, whereas smaller immune clusters were enriched in classical complement activation, IFNG and IL-1 responses. (Fig. 4c). Consistent with tissue damage, we found numerous small clusters in the airway, reflecting the presence of non-hematopoietic cells, including those containing multiple intermediate filament keratin genes, cell-cell adhesion claudin genes and surfactant genes. Notably, non-hematopoietic cell signatures in the airway were similar in content to those derived from in vitro SARS-CoV-2-infected primary lung epithelial cell lines (NHBE) $)^{12}$ (Supplementary Fig. 1d).

Given the large number of clusters including Mo/myeloid/MФ, we next examined these clusters in greater detail by altering the stringency of PPI clustering to further characterize unique myeloid lineage cells within each tissue compartment (Fig. 4d-f, Supplementary Table 4). Myeloid lineage-specific clusters were then compared to previously published gene signatures, including populations G1-G4 reported in BAL of COVID-19 patients ${ }^{29}$ (Supplementary Fig. 2a). In the blood, we found gene modules representative of common myeloid function (chemotaxis, proteolysis, etc.), as well as two independent Mo/myeloid subpopulations (Fig. 4d). Cluster 6 contained numerous markers highly reminiscent of classically activated blood Mo and exhibited significant overlap with the inflammatory G1 population, whereas cluster 1 was similar to IFN-activated MФs, CX3CR1 + synovial lining MФs (from arthritic mice) and alveolar MФs (AM) (Supplementary Fig. 2a).

In the lung (Fig. 4e), clusters 2, 3 and 6 overlapped with the G1 inflammatory Mo population and expressed a number of chemotaxis genes. A second population characteristic of AMs was also evident in the lung, defined by $C S F 2 R B$, the receptor for GM-CSF, a cytokine that regulates AM differentiation ${ }^{8,30,31}$. Further characterization of this population indicated significant expression of the coagulation system genes F5, FGG, FGL1, SERPINA and SERPINE2. Similarly, re-clustering of $\mathrm{Mo} / \mathrm{M} \Phi$ s/myeloid clusters from the airway revealed a population with hallmarks of inflammatory/M1 MФ (MARCO and multiple members of the complement cascade; cluster 7), and a second population of AMs (Fig. 4f) demonstrating significant overlap with the G3 and G4 populations (Supplementary Fig. 2a) $)^{29}$.

Characterization of myeloid populations in COVID-19 patients. The overlap between previously characterized BAL-defined gene signatures from COVID-19 patients ${ }^{29}$ and tissue-defined PPI clusters motivated us to evaluate these populations in greater detail by GSVA. Consistent with PPI clusters, the inflammatory-M $\Phi$ G1 population was increased in the blood (Supplementary Fig. 2b). The G1 and G1 \& G2 populations were increased in the lung, consistent with the expression of IFN and pro-inflammatory cytokines (Supplementary Fig. 2c). In the airway, the G2, G3, and G4 populations were significantly enriched indicating the presence of both pro-inflammatory MФs and AMs (Supplementary Fig. 2d-f). As a whole, we found that gene signatures of previously defined $\mathrm{Mo} / \mathrm{M} \Phi$ populations in COVID-19 BAL were dispersed among the blood, lung, and airway compartments.

Co-expression further delineates Mo/MФ gene expression profiles of COVID-19 patients. We next sought to identify the biology of the populations of $\mathrm{Mo} / \mathrm{M} \Phi$ in the tissue compartments in greater detail. We derived a set of 196 co-expressed Mo/myeloid genes and used them (Supplementary Fig. 3, Supplementary Table 5, see Methods) to probe heterogeneity in each tissue compartment (Fig. 5a). Notably, we found co-expression of 40 core genes between all compartments, which included complement, chemokine, and cytokine genes (Fig. 5b). In addition, there were 86 shared co-expressed genes in lung and blood, 57 in the lung and airway, and 61 in the airway and blood (Fig. 5b). To directly compare levels of these 196 co-expressed myeloid genes in each compartment, we normalized gene expression in each sample using 3 genes included in the core 40 genes, (FCGR1A, FCGR2A, FCGR2C) (Fig. 5c). Although many genes were not significantly different between compartments, numerous chemokines and cell surface markers (CCL2, CCL7, CCL8, CXCL10, CLEC4E, FCER1G) and inflammatory cytokines (IL1A and TNF) were enriched in the lung compared to the blood and airway. Furthermore, the complement genes $C 1 Q B, C 1 Q C$, and $C 2$ were increased in the lung compared to the blood, but not changed between the lung and the airway. Altogether, these normalized gene expression results suggested that expression of inflammatory mediators was increased in SARS-CoV2-infected lung over the other compartments and in the airway compared to the blood.

To determine the function and nature of these myeloid populations, we compared them to previously published myeloid signatures (Fig. 5d, Supplementary Table 6) ${ }^{29,35-38}$. The population increased in the blood (A1) was predominantly characterized by features of AMs, M1 and M2 MФs, pro-inflammatory MФs with potential to infiltrate tissue, and the inflammatory $M \Phi$ G1 population. The A1 population also exhibited features of inflamed murine residential, interstitial MФs. The myeloid cell population increased in COVID lung (A2) was most similar 
a

PBMC-CoV2 Myeloid A1

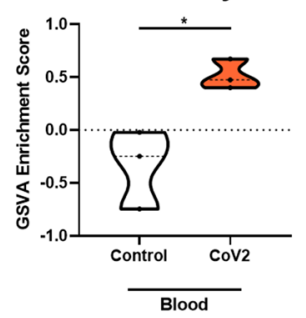

Lung-CoV2 Myeloid A2

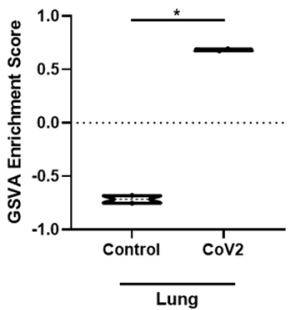

b

BAL-CoV2 Myeloid A3

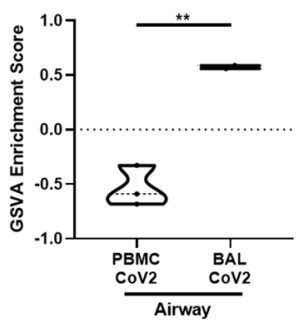

Overlap of CoV2 Co-expressed Genes

PBMC

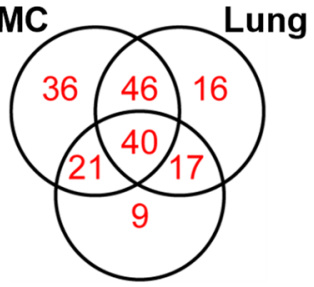

BAL
C

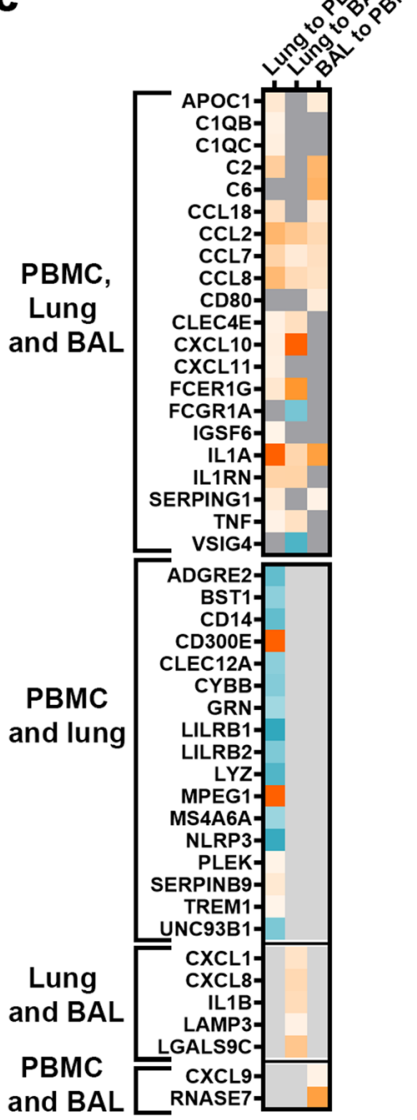

d

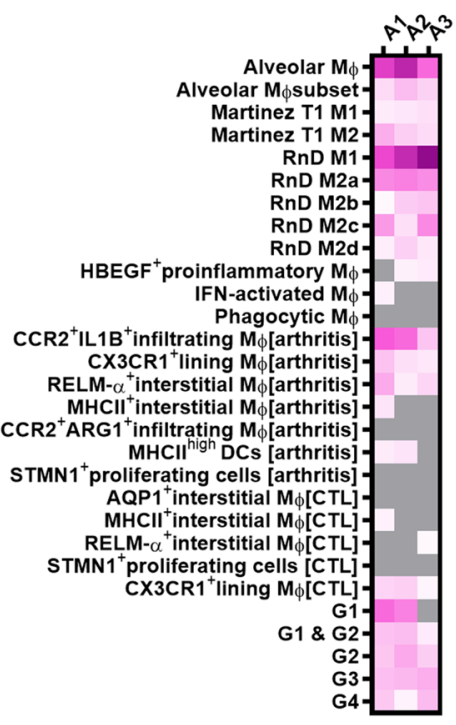

e $f$
0

0
0
0

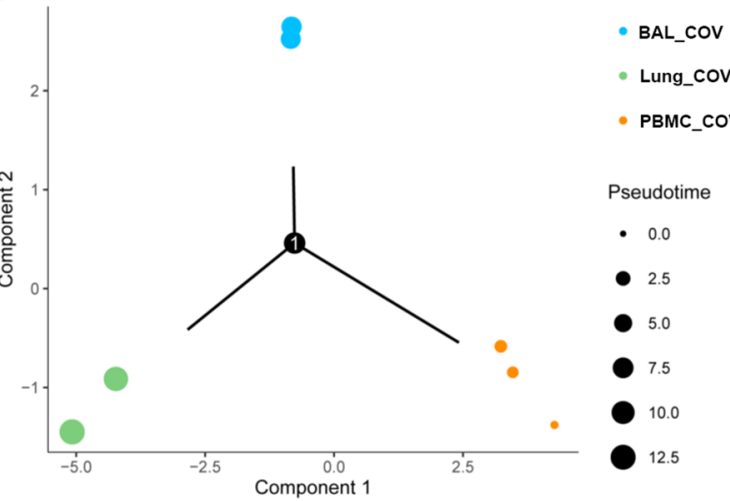

Figure 5. Different co-expression-derived myeloid populations are found in blood, lung, and airway of COVID-19 patients. (a) GSVA enrichment of myeloid subpopulations increased in COVID-19 blood (A1), lung (A2), and airway (A3). (b) Venn Diagram of the gene overlap between myeloid subpopulations A1-A3. (c) Comparison of normalized $\log _{2}$ fold change expression values of genes defining A1-A3. Expression values for each sample in each comparison were normalized by the mean of the $\log _{2}$ fold change expression of FCGR1A, FCGR2A, and FCGR2C. Significant comparisons are displayed by Hedge's G effect size. (d,e) Characterization of A1-A3 by enrichment of previously described myeloid populations (d) (Supplementary Table 3,6) and PBMC, lung, and BAL myeloid metaclusters from Fig. 4d-f (e). Fisher's Exact Test was used to calculate overlap between transcriptomic signatures and significant overlaps $(p<0.05)$ are shown as the negative logarithm of the $p$ value. (f) Trajectory analysis using expression of 621 genes (196 myeloid-specific genes used in a,b +425 additional myeloid genes shown in Supplementary Table 5) in the blood, lung, and airway compartments. Colors represent sample identity and size represents pseudotime distance along the trajectory. Generated using GraphPad Prism v8.4.2 (www.graphpad.com) and the R package Monocle v2.14.0 $0^{32-34}$. 
to pro-fibrotic AMs, M1 MФs, M2 MФs, blood-derived infiltrating MФs, and the inflammatory Mo G1 population. A2 was also marked by additional AM-specific genes, contributing to the observed overlap with the other two compartments. However, overlap between A2 and the G4 AM signature was relatively decreased, suggesting that the lung AMs are more similar to those found in pulmonary fibrosis ${ }^{35}$. Finally, the population increased in the airway (A3) similarly exhibited characteristics of AMs, M1 and M2 MФs, and pro-inflammatory MФs that have infiltrated into the tissue compartment (Fig. 5d). Of note, the airway A3 population was not similar to the previously described BAL-derived inflammatory $\mathrm{M} \Phi \mathrm{G1}$ population ${ }^{29}$.

We also evaluated the overlap between the Mo/MФ A1-A3 gene clusters and those identified using PPI clustering (Fig. 4) (Fig. 5e, Supplementary Table 6). Interestingly, the CD33 ${ }^{+}$pathogenic population (PPI-derived PBMC Myeloid Cluster 1) was most strongly enriched in the blood, but was also increased in the other compartments. All compartments were characterized by strong enrichment of pro-inflammatory Mo (PBMC Myeloid Cluster 6, Lung Myeloid Clusters 3 and 6, and BAL Myeloid Cluster 7), although A3 exhibited some differences in these populations compared to A1 and A2. Additionally, this comparison suggested enrichment of AMs in all three compartments; however, upon examination of the specific overlapping gene transcripts, the observed enrichment in blood A1 was primarily related to the presence of non-AM-specific myeloid genes. Finally, numerous common activators and functions of PPI-derived clusters were enriched uniformly across A1, A2, and A3, providing further evidence for pro-inflammatory activity of myeloid cell populations in COVID-19 blood and tissue compartments.

We used trajectory analysis to understand potential transitions of $\mathrm{Mo} / \mathrm{M} \Phi$ in various tissue compartments. We based this on the normalized 196 myeloid-cell specific genes, as well as 425 additional normalized genes that could be important in Mo/myeloid/M $\Phi$ cell differentiation, reflective of chemotaxis, IFN, and metabolism genes (Supplementary Table 6). This analysis suggested a branch point of differentiation of Mo/M $\Phi$ between blood and lung, with some blood $\mathrm{Mo} / \mathrm{M} \Phi$ differentiating directly to airway cells and others to lung cells in a more protracted manner as indicated by pseudotime (Fig. 5f).

Analysis of the biologic activities of myeloid subpopulations. To focus on functional distinctions among the co-expressed myeloid populations in the blood, lung, and airway compartments (A1-A3), we utilized linear regression analyses between GSVA scores for A1-A3 and scores for metabolic, functional, and signaling pathways (Fig. 6, Supplementary Fig. 4, Supplementary Table 3). Blood A1 was significantly correlated with glycolysis, the NLRP3 inflammasome, and the classical and lectin-induced complement pathways. In lung A2, there were no significant correlations detected with metabolism, but this population was significantly correlated with the NLRP3 inflammasome and the alternative complement pathway. Finally, airway A3 was positively correlated with OXPHOS, the classical complement pathway, and TNF signaling and negatively correlated with apoptosis. Overall, these results delineated the heterogeneity in metabolic and inflammatory pathways among myeloid cells enriched in the blood, lung, and airway of COVID-19 patients.

Pathway and upstream regulator analysis inform tissue-specific drug discovery for treatment of COVID-19. To understand the biology of SARS-CoV2-infected patients in greater detail, we conducted pathway analysis on DEGs from the 3 compartments using IPA canonical signaling pathway and upstream regulator (UPR) analysis functions (Fig. 7). In general, IFN signaling, the inflammasome, and other components of anti-viral, innate immunity were reflected by disease state gene expression profiles compared to healthy controls (Fig. 7a). In addition, metabolic pathways including OXPHOS and glycolysis were significantly increased in the blood of COVID-19 patients compared to controls.

UPRs predicted to drive the responses in each compartment indicated uniform involvement of inflammatory cytokines, with Type I IFN regulation dominant in the SARS-CoV2-infected lung (Fig. 7b). Notable UPRs of COVID-19 blood included IFNA, IFNG, multiple growth factors and ligands, HIF1A, CSF1 and CSF2. Evidence of inflammatory cytokine signaling by IL17 and IL36A was predicted in COVID-19 lung and airway compartments. Whereas the airway DEG profile indicated regulation by both inflammatory and inhibitory cytokines, the COVID-19 lung UPRs were markedly inflammatory, including, NFkB, IL12, TNF, IL1B, and multiple Type I IFNs. These proinflammatory drivers were consistent in each individual lung which we analyzed separately because of the apparent heterogeneity between the lung samples (Supplementary Fig. 5).

IPA analysis was also employed to predict drugs that might interfere with COVID-19 inflammation (Fig. 7b, Supplementary Table 7). Of note, neutralizers of IL17, IL6, IL1, IFNA, IFNG, and TNF were predicted as antagonists of COVID-19 biology. Corticosteroids were predicted to revert the gene expression profile in the SARSCoV-2-infected lung, but were predicted as UPRs of COVID-19 blood, which may indicate that the patients from whom blood was collected had been treated with corticosteroids rather than indicating that these agents were driving disease pathology. Chloroquine (CQ) and hydroxychloroquine (HCQ) were additionally predicted to revert the COVID-19 transcription profile in the lung, which may point to their potential utility as treatment options. A number of drugs matched to unique targetable pathways in the lung, including NFKB pathway inhibitors and neutralizers of the TNF family; however, some drugs also targeted pathways shared by both the lung and airway, including JAK inhibitors. In the BAL-CoV2 vs. PBMC-CoV2 IPA comparison, several drugs were matched to UPRs with a negative Z-score, which provided additional therapeutic options directed towards the blood of patients with COVID-19, given that molecules targeting downregulated or inhibited UPRs are molecules that could normalize the PBMC-CoV2 gene signature. As such, several possible therapeutics arose from this analysis to target COVID-19 blood, including ustekinumab, targeting the IL12/23 signaling pathway and lenalidomide, all of which has immunosuppressive effects. In addition, IGF1R inhibitors, EGFR inhibitors, VEGFR inhibitors, and AKT inhibitors were among the compounds predicted to target COVID-19 PBMCs. 
Metabolism

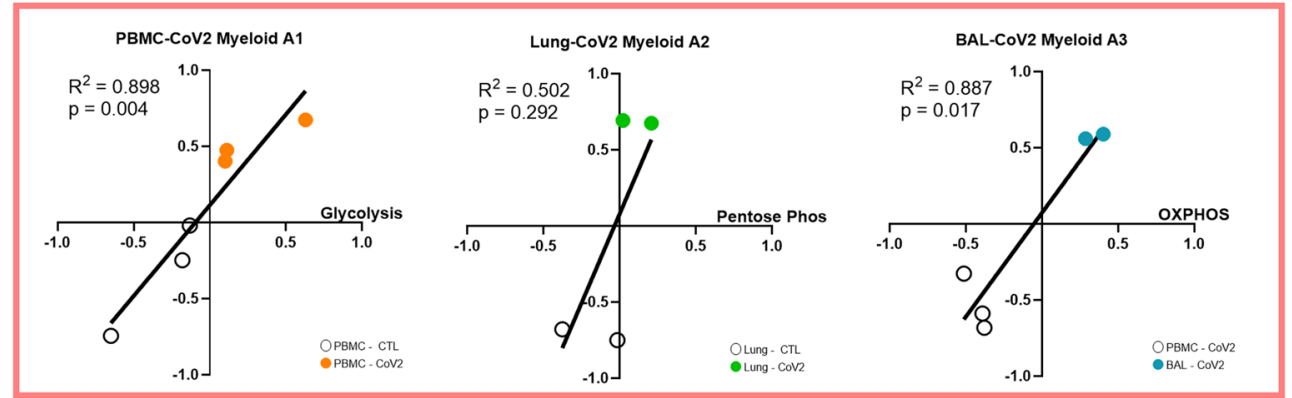

\section{Inflammasome}

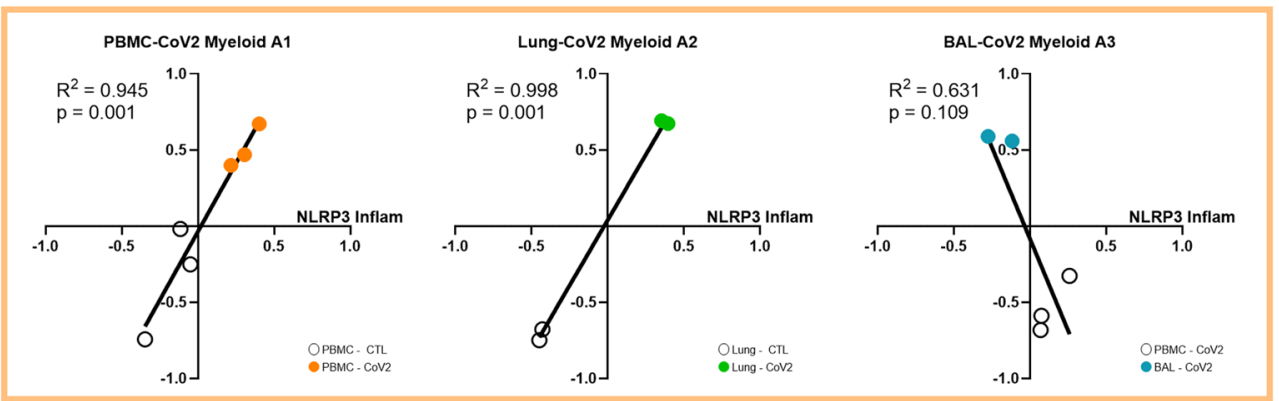

\section{Complement}

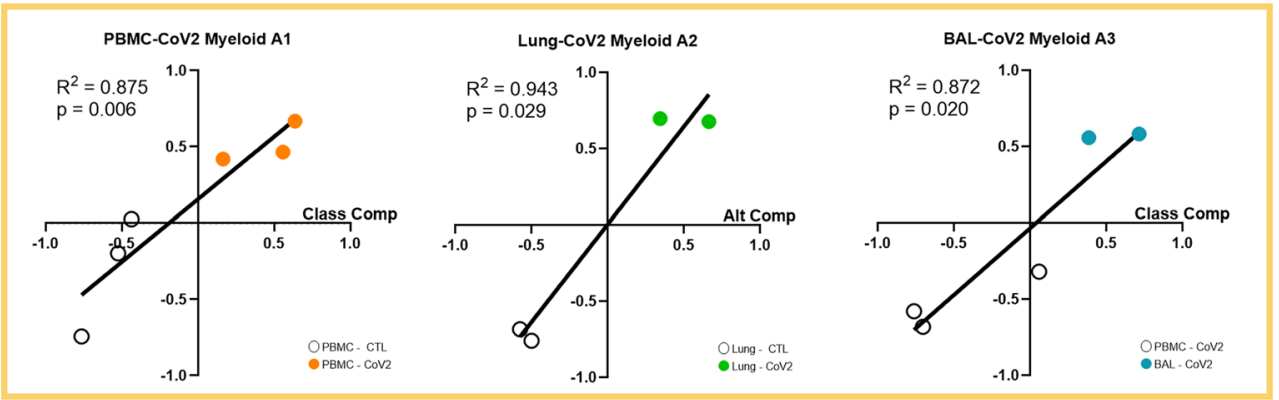

\section{Apoptosis}

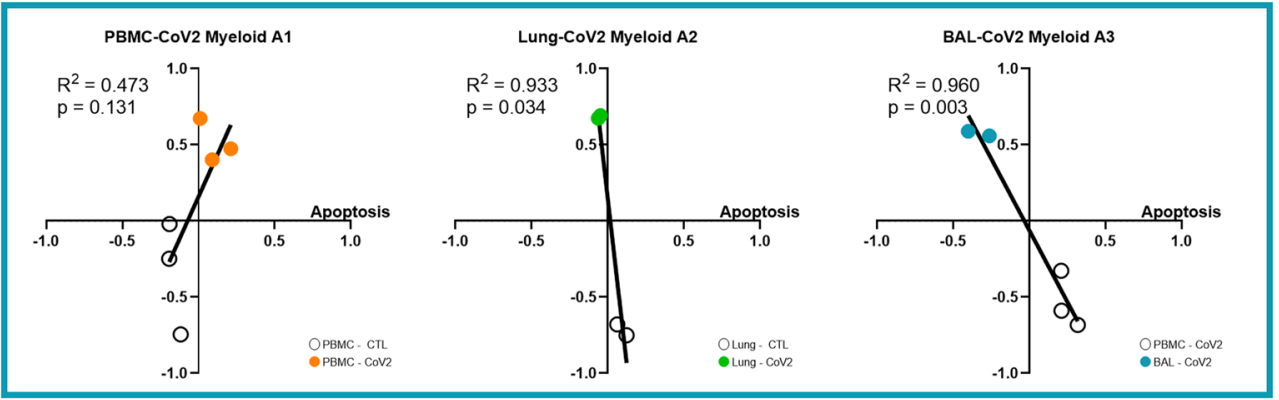

\section{TNF Signaling}
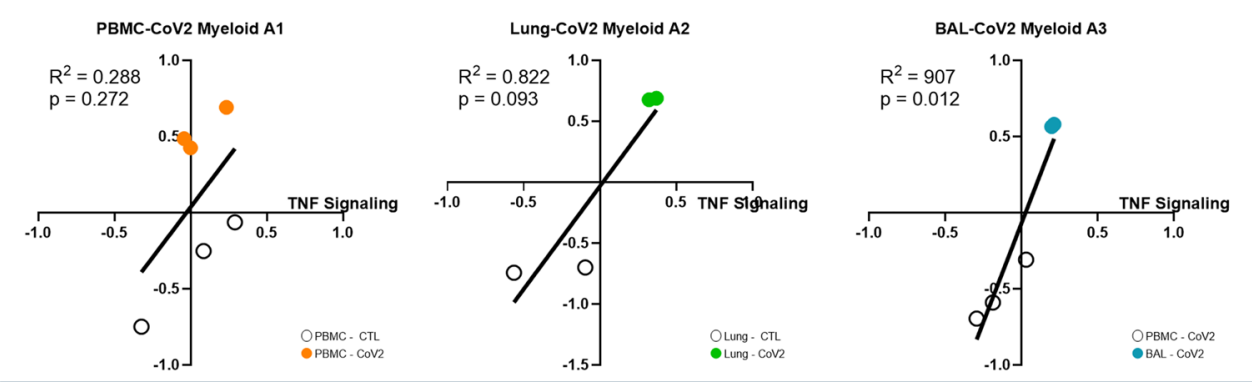

Figure 6. Analysis of biological activities of myeloid subpopulations. Linear regression between GSVA scores for each of the tissue-specific myeloid populations (A1-A3) and metabolism, NLRP3 Inflammasome, complement, apoptosis, and TNF signaling. Generated using GraphPad Prism v8.4.2 (www.graphpad.com). 
No specific drugs were predicted to target all three tissue compartments, but each compartment was driven by inflammatory cytokines.

Another means to predict possible drug targets is by employing connectivity scoring with drug-related gene expression profiles using the perturbagen CMAP database within CLUE (Supplementary Table 7, see Methods). Although CLUE-predicted drugs tended to differ from those predicted by IPA or those matched to IPApredicted UPRs, there were some overlapping mechanisms, including inhibition of AKT, angiogenesis, CDK, EGFR, FLT3, HSP, JAK, and mTOR. IPA-predicted drugs that were unique from connectivity-predicted drugs tended to capture more cytokine and lymphocyte biology, including inhibitors of IL1, IL6, IL17, TNF, type I and II interferon, CD40LG, CD38, and CD19, among other cytokines and immune cell-specific markers. Overall, our gene expression-based analysis of SARS-CoV2-infected blood and tissue compartments indicated several existing treatment options that could be considered as candidates to treat COVID-19.

\section{Discussion}

Multiple orthogonal bioinformatics approaches were employed to analyze DEG profiles from the blood, lung, and airway of COVID-19 patients and revealed the dynamic nature of the inflammatory response to SARSCoV-2 and possible points of therapeutic intervention. In the blood, we saw evidence of myeloid cell activation, lymphopenia, and elevation of plasma cells, as has been shown by both standard cell counts, flow cytometry and gene expression analysis ${ }^{39}$. In the lungs, we found increased gene signatures of additional myeloid cell types including granulocytes, infiltrating inflammatory Mo, and AMs as well as the presence of non-activated T, B, and NK cells. Furthermore, inflammation in the lung tissue was enhanced by the greater presence of IFNs and more pro-inflammatory cytokines than observed in the blood. Finally, in the airway we found evidence of blood and $A M$-derived inflammatory and regulatory MФs, and non-hematopoietic lung tissue cells accompanied by expression of SARS-CoV-2 receptors, and alarmins, indicative of viral infection and damage to the lung and consistent with previous reports of detection of SARS-CoV2 in BAL fluid ${ }^{11,40}$. Together these findings suggest a systemic, but compartmentalized immune/inflammatory response with specific signs of cellular activation in blood, lung and airway. This has informed a more comprehensive and integrated model of the nature of the local and systemic host response to SARS-CoV2.

The predominant populations of immune cells we found to be enriched and activated in COVID-19 patients were myeloid cells and, in particular, subsets of inflammatory Mo and $M \Phi s$, which differed between the blood, lung, and airway compartments. In the peripheral blood, we found significant enrichment of $M \Phi$ s, including classically activated inflammatory M1 MФs as well as a CD33 ${ }^{+}$myeloid subset, which appeared to be an M2 population reminiscent of previously characterized IFN-activated MФs, AMs, and MDSCs, indicative of a potential regulatory population induced by stimuli arising from the SARS-CoV2-infected lung. Myeloid cells enriched in the blood of COVID-19 patients were also highly correlated with gene signatures of metabolic pathways (Glycolysis, Pentose Phosphate Pathway, and TCA cycle) indicative of pro-inflammatory M1 MФs ${ }^{41}$.

The lung tissue was enriched in gene signatures of Mo/MФs as well as other myeloid cells including two populations of granulocytes, neutrophils and LDGs. Increases in blood neutrophils have been found to be associated with poor disease outcome in COVID-19 patients and it has been proposed that the formation of neutrophil extracellular traps (NETs) contributes to increased risk of death from SARS-CoV2 infection ${ }^{42-44}$. In addition, recent reports have characterized populations of dysregulated neutrophils expressing pro-inflammatory or suppressive markers derived from scRNA-seq of COVID-19 patient PBMCs, which were positively correlated with disease severity ${ }^{19,20}$. We found that these populations were also increased in SARS-CoV2 infected lung tissue and, therefore, suggest that they may contribute to lung pathology. Although LDGs have not previously been reported in the COVID-19 lung, in comparison to neutrophils, they exhibit an enhanced capacity to produce Type I IFNs and form NETs and therefore, may have an even greater impact on disease progression ${ }^{45}$.

$\mathrm{Mo} / \mathrm{M} \Phi$ subsets in the lung of COVID-19 patients were characterized as infiltrating inflammatory Mo and activated AMs, which exhibited a mixed metabolic status suggestive of different states of activation. Infiltrating Mo from the peripheral blood appeared to be further activated in the lung tissue as evidenced by enhanced expression of markers of highly inflammatory Mo previously characterized in severe COVID-19 $\mathrm{cases}^{29}$. The AM population enriched in COVID lung tissue clustered with genes involved in the coagulation system, which is consistent with observations of procoagulant AM activity in COVID-19 and in ARDS ${ }^{46}$. As pulmonary thrombosis has been associated with poor clinical outcomes in COVID-19 patients, this result suggests that activated AMs in SARS-CoV2-infected lung tissue may be involved in facilitating a pro-thrombotic status, and thereby, contribute to poor disease outcome $\mathrm{e}^{47}$. Finally, although not statistically significant, we noted a trend toward an increase in platelets specifically in the COVID-19 lung, suggesting that they may also contribute to thrombosis in some patients.

In the airway, we detected gene signatures of various post-activated $M \Phi$ subsets including inflammatory $\mathrm{M} 1$ MФs, alternatively activated M2 MФs, and activated AMs. Expression of myeloid cell genes in the airway also correlated with a signature of oxidative metabolism, which is characteristic of M2 macrophages and typically associated with control of tissue damage $e^{41}$. However, in the context of pulmonary infection, polarization of AMs toward an anti-inflammatory M2 phenotype was found to promote continued inflammation, suggesting that these MФs may not be effective at resolving anti-viral immunity ${ }^{48}$.

In addition to myeloid cells, inflammatory mediators from the virally infected lung typically promote migration and activation of NK cells and adaptive immune cells including $\mathrm{T}$ and $\mathrm{B}$ cells ${ }^{8}$. We found significant deficiencies in gene signatures of T cells and cytotoxic CD8 and NK cells, consistent with clinical evidence of lymphopenia in the peripheral blood and airway of COVID-19 patients ${ }^{49-52}$. In contrast to T and NK cells, we observed increased evidence of B cell activation through CD40/CD40L and an increased plasma cell signature in the blood of COVID-19 patients. This result suggests that COVID-19 patients are able to mount an antibody-mediated 
immune response. However, whether a virus-specific antibody response is beneficial to recovery from SARS$\mathrm{CoV} 2$ infection is unclear ${ }^{53}$. Low quality, low affinity antibody responses to SARS-CoV have been found to promote lung injury in some patients, although it is unknown if this occurs in SARS-CoV2 infected individuals ${ }^{54,55}$.

The contents of the airway as assessed through the BAL fluid, act as a window into events in the alveoli and airways and can be used to understand what is happening in the infected tissue that is separate from the interstitium of the lung ${ }^{56,57}$. We found increased enrichment of lung epithelial cells in the airway of COVID-19 patients, suggesting that SARS-CoV2 infection of alveolar cells together with localized inflammation as a result of enhanced myeloid cell infiltration promote significant damage to the alveoli and result in affected cells being sloughed into the airway. Furthermore, the lack of cytotoxic cells and thus, the inability to clear these virusinfected lung epithelial cells in the airway likely accounts for the increased presence of post-activated MФs and high expression of pro-inflammatory IL-1 family members we observed in the BAL of COVID-19 patients. Importantly, these results suggest that sampling of BAL may provide an important mechanism to evaluate the impact of SARS-CoV2 infection.

DEGs from COVID-19 patients were enriched in IGS, complement pathways, inflammatory cytokines and the inflammasome, which would be expected to activate Mo/MФ populations in the blood, lung, and airway of COVID-19 patients and initiate a robust and systemic response to infection. In particular, our results support the conclusion that IL-1 family-mediated inflammation plays a critical role in COVID-19 pathogenesis. However, pro-inflammatory genes identified in a GWAS study as contributing to COVID-19 inflammation, including CCR2, CCR3, CXCR6, and MTA2B, were not significantly different from controls in our lung dataset ${ }^{58}$. Thus, in contrast to previous characterizations of both SARS-CoV and SARS-CoV2 infection, our analysis did not indicate the presence of overwhelming pro-inflammatory cytokine production or "cytokine storm" in COVID-19 patients ${ }^{39,59}$. Rather, our data suggests that the increased numbers, overactivation, and potentially heightened pathogenicity of monocyte/MФ populations are the main drivers of the dysregulated inflammatory response and resulting tissue damage in COVID-19 patients.

In the absence of proven antiviral treatment and/or a SARS-CoV2-specific vaccine, disease management is reliant upon supportive care and therapeutics capable of limiting the severity of clinical manifestations. Using empiric evidence as a guide, the current approach has been successful in identifying "actionable" points of intervention in an unbiased manner and in spite of formidable patient heterogeneity. Analyses presented here support several recent reports highlighting COVID-19 infection-related increases in inflammatory cytokines, particularly IL6 and TNF, both of which function as predictors of poor prognosis ${ }^{60,61}$, as well as complement activation ${ }^{42,62,63}$. Accordingly, anti-IL6 therapies including sarilumab, tocilizumab and clazakizumab, as well as biologics targeting terminal components of the complement cascade, such as eculizumab and ravulizumab, are in various clinical trial phases for treating COVID-19-associated pneumonia. Candidate TNF blockers such as adalimumab, etanercept and many others, represent additional options for inhibiting deleterious pro-inflammatory signaling. However, most showed patient heterogeneity, suggesting a requirement to identify the specific cytokine profile in each patient in order to offer personalized treatment. Our analyses also point to the likely involvement of pro-inflammatory IL1 family members especially in the lung, suggesting anti-IL1 family interventions, including canakinumab and anakinra, may be effective in preventing acute lung injury.

This analysis also establishes the predominance of inflammatory Mo/myeloid lineage cells in driving disease pathology and suggests therapies effective at blocking myeloid cell recruitment or forcing repolarization may prevent disease progression. CCL5 (RANTES) is a potent leukocyte chemoattractant that interacts with multiple receptors, including CCR1 (upregulated in the blood, lung and airway), and CCR5 (upregulated in the airway). Disruption of the CCR5-CCL5 axis was recently tested using the CCR5 neutralizing monoclonal antibody leronlimab in a small compassionate use trial with promising preliminary results ${ }^{64}$.

It has also been observed that COVID-19 may predispose patients to thromboembolic disease ${ }^{65,66}$. Indeed, the gene expression analyses presented here showing altered expression of coagulation factors and fibrinogen genes suggests dysfunction within the intrinsic clotting pathway. These findings, together with evidence of excessive inflammation, complement activation and the involvement of LDGs in lung inflammation, may contribute to the systemic coagulation underlying the remarkably high incidence of thrombotic complications observed in severely ill patients, thereby reinforcing recommendations to apply pharmacological anti-thrombotic medications.

Finally, there has been much recent discussion concerning the use of anti-rheumatic drugs for managing COVID-19. In fact, CQ was one compound predicted as a UPR with potential phenotype-reversing properties. In vitro experiments examining the anti-viral properties of CQ and its derivative HCQ were effective in limiting viral load; however, the efficacy of these drugs in clinical trials has been less clear ${ }^{67}$. Questions about drug timing, dosage and adverse events have all called into question the use of these drugs for COVID-19 patients. Despite a recent report showing no negative connectivity between the gene signatures of SARS-CoV2 infection and HCQ treatment ${ }^{40}$, IPA predicted a role of anti-malarials as limiting the function of intracellular TLRs in the lung and also as a direct negative UPR of gene expression abnormalities in the lung, suggesting a role in controlling COVID-19 inflammation and not viral replication. Further clinical testing may be necessary to test this possible utility.

By comparing the transcriptomic profile of the blood, lung, and airway in COVID-19 patients, a model of the systemic pathogenic response to SARS-CoV2 infection has emerged (Fig. 8). SARS-CoV2 infection leads to systemic Mo/MФ activation, likely as a result of the release of pro-inflammatory mediators from infected cells. Infiltration of immune cells into the lung tissue and alveolus, in particular, neutrophils, LDGs, and pathogenic $\mathrm{Mo} / \mathrm{M} \Phi$ populations promotes a cycle of inflammatory mediator release and further myeloid cell activation, which exacerbates inflammation in the lung and leads to tissue damage. The local release of complement components and clotting factors by infiltrating $\mathrm{Mo} / \mathrm{M} \Phi$ may contribute to both inflammation and thrombotic events. As disease progresses, increased infiltration of pro-inflammatory immune cells, release of inflammatory mediators, and damage to the infected alveolus is reflected by the presence of Mo/M $\Phi$ cells and lung epithelial 
a Canonical Signaling Pathways

PBMC-CoV2 vs. PBMC-CTL

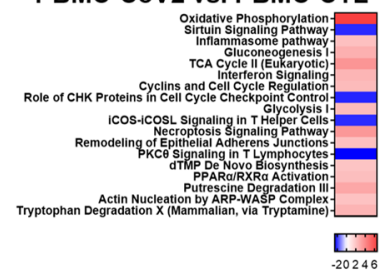

Activation Z-Score

$p<0.01$
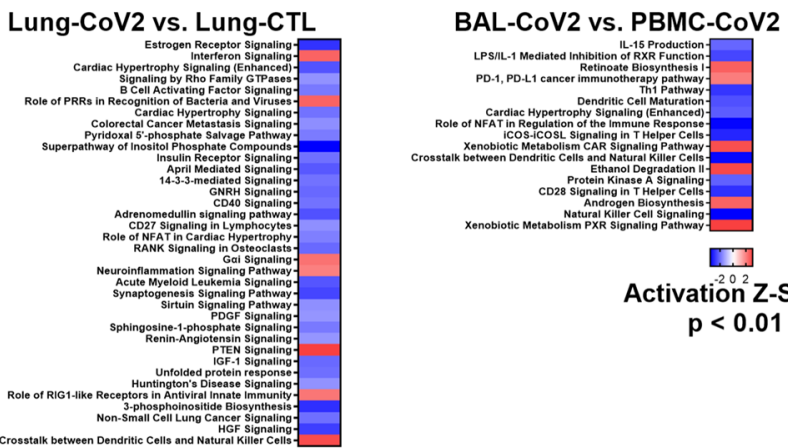

Activation Z-Score $p<0.01$

\section{b Upstream Regulators}

PBMC-CoV2 vs. PBMC-CTL
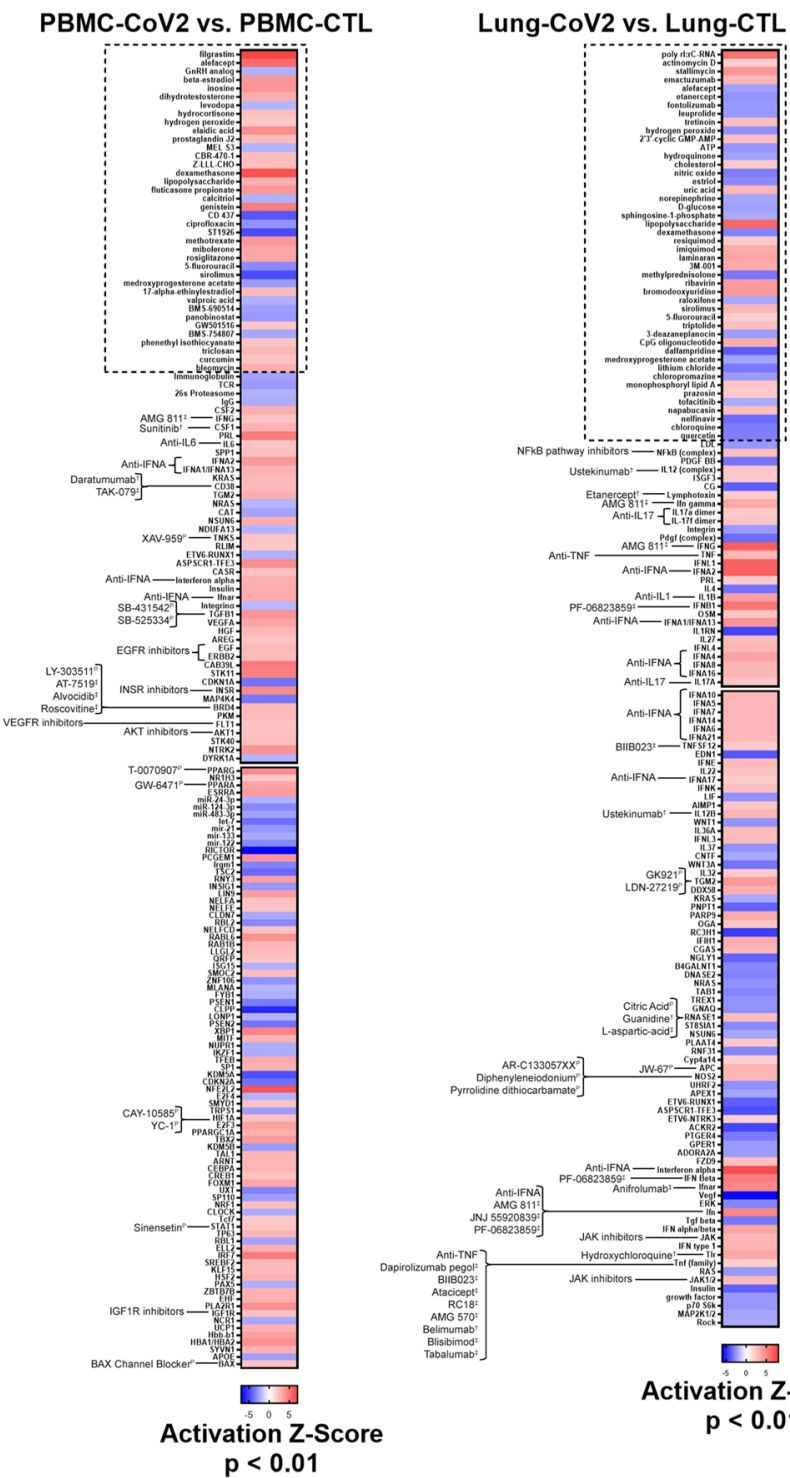

Activation Z-Score

$p<0.01$

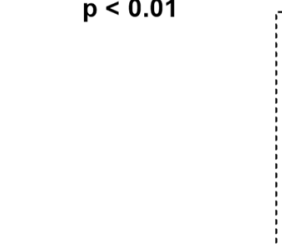

BAL-CoV2 vs. PBMC-CoV2
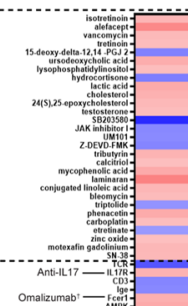

$$
\text { . }
$$

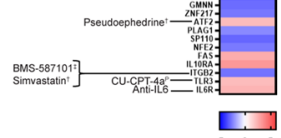

Activation Z-Score

$\mathrm{p}<0.01$

Activation Z-Score

$\mathrm{p}<0.01$

Figure 7. Pathway Analysis of SARS-CoV-2 blood, lung, and airway. DEGs from each SARS-CoV-2 blood or tissue pairwise comparison were uploaded into IPA (Qiagen Inc., https://www.qiagenbioinformatics.com/ products/ingenuity-pathway-analysis) and canonical signaling pathway (a) and upstream regulator (b) analyses were performed. Heatmaps represent significant results by Activation Z-Score $\geq|2|$ and overlap $p$-value $<0.01$. The boxes with the dotted outline separate drugs that were predicted as upstream regulators from pathway molecules and complexes. The remaining, significant upstream regulators were matched with drugs with known antagonistic targeting mechanisms. The top 150 UPRs in the lung are shown in (b) and the remaining are in Supplementary Fig. 5. Specific drugs for particular drug families (e.g., Anti-IL17) are found in Supplementary Table 7. ${ }^{\dagger}$ : FDA-approved. ${ }^{*}$ : Drug in development/clinical trials. ${ }^{\text {P: }}$ Preclinical. 


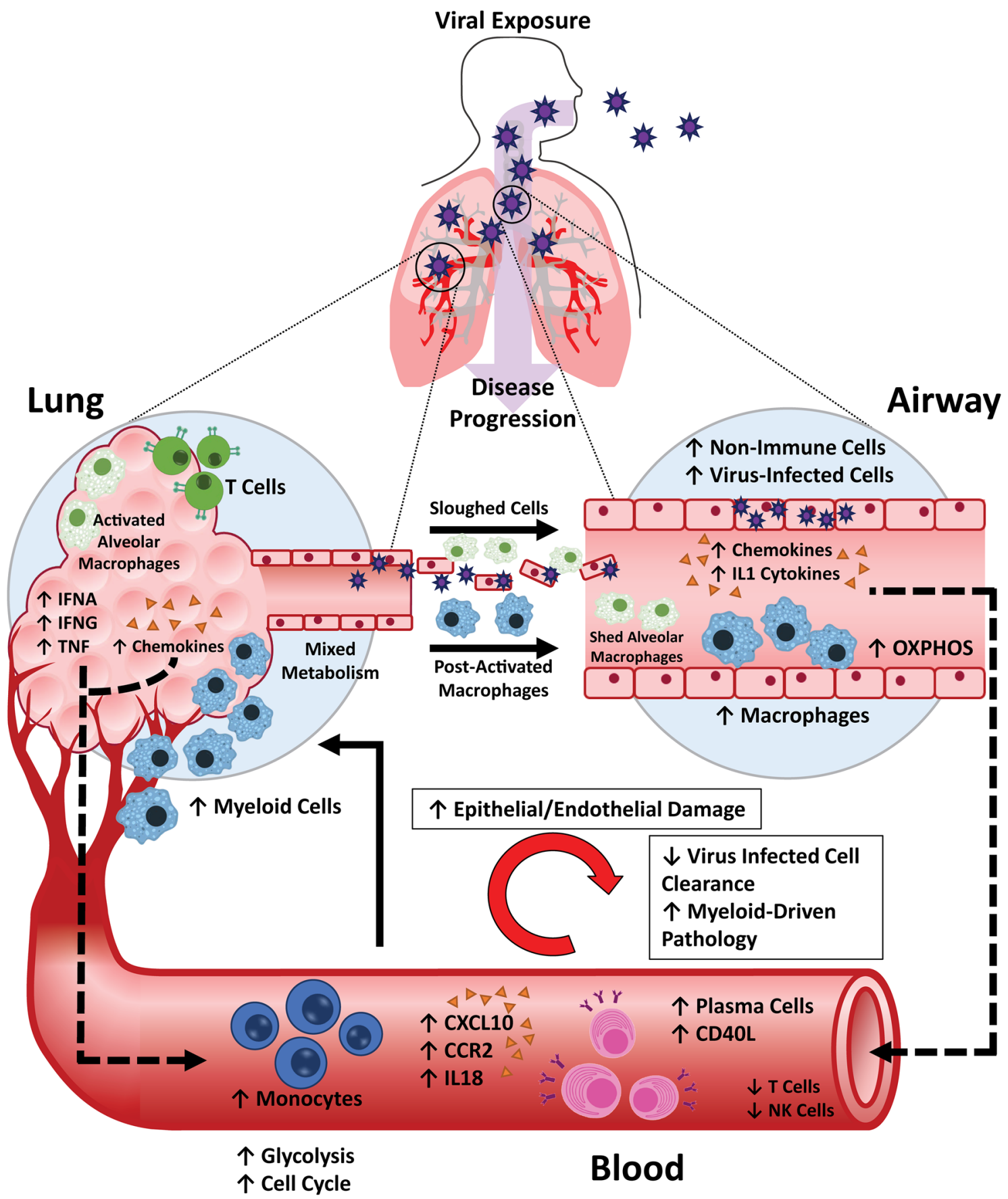

Figure 8. Graphical model of COVID-19 pathogenesis. Proposed model of the inflammatory response to SARS-CoV2 infection in three compartments: the blood, lung, and airway generated using Microsoft PowerPoint version 19.0 and Adobe Illustrator version 25.0.

cells in the airway ${ }^{56}$. Furthermore, evidence of both Mo-derived inflammatory MФs and AMs in the airway compartment suggests that myeloid cell populations from both the blood and the lung tissue are present in the BAL. The accumulation of virus-infected cells and release of alarmins in the airway may not only reflect ongoing infection, but also promote inflammation and prevent resolution of the infection and foster the continuation of the innate immune response. Therefore, sampling the BAL fluid seems to be an effective strategy to monitor tissue inflammation and damage in COVID-19 patients.

As SARS-CoV2 continues to propagate, viral clearance is impaired by a lack of cytotoxic CD8 T cells and NK cells. This is consistent with MAS occurring in other settings, in which defects in cytotoxic activity of CD8 T cells and NK cells result in enhanced innate immune cell activation and intensified production of pro-inflammatory cytokines, many of which were also expressed in COVID-19 patients $^{9,10}$. Thus, we propose that the lack of activated CD8 T cells and NK cells and subsequent failure to clear virus-infected cells, is a major contributor to the $\mathrm{M} \Phi$-driven pathologic response to SARS-CoV2 observed in COVID-19 patients. 
In order to develop a model of SARS-CoV2 infection, we have utilized multiple orthogonal approaches to analyze gene expression from COVID-19 patients, but also acknowledge the limitations of this data. For example, we were only able to analyze 2-3 samples per experimental condition and this limited the statistical power of each of our bioinformatics techniques. In addition, the low number of samples meant that heterogeneity among patients in any given cohort had an increased impact on the overall outcome. One possible reason for this intracohort heterogeneity is that the patients may have exhibited varying levels of disease severity and, unfortunately, we did not have access to clinical information. These points highlight the need for additional studies on more patients, preferably accounting for differences in demographic information and disease status, in order to increase the power of downstream analyses.

In conclusion, transcriptomic analysis has contributed critical insights into the pathogenesis of COVID-19. Diffuse Mo/MФ activation is the likely primary driver of clinical pathology. Therefore, this work provides a rationale for placing greater focus on the detrimental effects of exaggerated activation of pathogenic Mo/MФs and for targeting these populations as an effective treatment strategy for COVID-19 patients.

\section{Methods}

Ethics statement. Publicly available data sets used in this study are listed in Supplementary Table 1. For each dataset, all patient samples were collected in adherence to local regulations and after obtaining institutional review board approved informed consent. The study corresponding to accession number CRA002309 was approved by the Ethics Committee of the Zhongnan Hospital of Wuhan University. The study corresponding to accession number GSE147505 was approved by the institutional review board at the Icahn School of Medicine at Mount Sinai under protocol HS\#12-00145.

Read quality, trimming, mapping and summarization. RNA-seq data were processed using a consistent workflow using FASTQC, Trimmomatic, STAR, Sambamba, and featureCounts. As described below SRA files were downloaded and converted into FASTQ format using SRA toolkit. Read ends and adapters were trimmed with Trimmomatic (v0.38) using a sliding window, ilmnclip, and headcrop filters. Both datasets were head cropped at $6 \mathrm{bp}$ and adapters were removed before read alignment. Reads were mapped to the human reference genome hg38 using STAR, and the sam files were converted to sorted bam files using Sambamba. Read counts were summarized using the featureCounts function of the Subread package (v1.61.)

The RNA-seq tools are all free, open source programs available at the following web addresses.

SRA toolkit-https://github.com/ncbi/sra-tools

FastQC-https://www.bioinformatics.babraham.ac.uk/projects/fastqc/

Trimmomatic-http://www.usadellab.org/cms/?page=trimmomatic

STAR—https://github.com/alexdobin/STAR

http://labshare.cshl.edu/shares/gingeraslab/www-data/dobin/STAR/STAR.posix/doc/STARmanual.pdf

Sambamba-http://lomereiter.github.io/sambamba/

FeatureCounts-http://subread.sourceforge.net/

Differential gene expression and gene set enrichment analysis. The DESeq2 workflow was used for differential expression analysis. Comparisons were made between control PBMCs and PBMCs from COVID19 patients (PBMC-CTL vs PBMC-CoV2) and control lung tissue and lung tissue from COVID-19 patients (Lung-CTL vs Lung-CoV2). Since no corresponding control BAL samples were available for the COVID-19 BAL samples, we compared BAL samples from COVID-19 patients to COVID-19 PBMC (PBMC-CoV2 vs BALCoV2). This was possible because these samples were analyzed on the same platform, run at the same time, and it was done understanding the limitations of this analysis. We also compared normal BAL to BAL of asthmatic individuals to identify genes unrelated to COVID-19 (PRJNA434133).

Two technical replicates were included for BAL cohort, and 4 technical replicates were included for postmortem lung samples. The replicates were collapsed and averaged into one using collapsereplicates function from DESeq2 package. The genes with low expression (i.e. genes with very few reads) were removed by filtration. The filtered raw counts were normalized using the DESeq method and differentially expressed genes were determined by FDR $<0.2^{68}$. Counts were then $\log 2$ transformed and used for downstream analyses (Supplementary Table 2).

Gene set variation analysis (GSVA). The $\mathrm{GSVA}^{69}$ (V1.25.0) software package is an open source package available from R/Bioconductor and was used as a non-parametric, unsupervised method for estimating the variation of pre-defined gene sets in patient and control samples of microarray and RNA-seq expression data sets (www.bioconductor.org/packages/release/bioc/html/GSVA.html). The inputs for the GSVA algorithm were a gene expression matrix of $\log _{2}$ expression values for pre-defined gene sets (Supplementary Table 3). All genes within a gene set were evaluated if the interquartile range (IQR) of their expression across the samples was greater than 0. Enrichment scores (GSVA scores) were calculated non-parametrically using a Kolmogorov Smirnoff (KS)-like random walk statistic and a negative value for a particular sample and gene set, indicating that the gene set has a lower expression than the same gene set with a positive value. The enrichment scores (ES) were the largest positive and negative random walk deviations from zero, respectively, for a particular sample and gene set. The positive and negative ES for a particular gene set depend on the expression levels of the genes that form the pre-defined gene set. GSVA calculates enrichment scores using the $\log _{2}$ expression values for a group of genes in each SARS-CoV2 patient and healthy control and normalizes these scores between -1 (no enrichment) and +1 (enriched). Welch's $t$ test was used to calculate the significance of the gene sets between the cohorts. Significant enrichment of gene sets was determined by $p$ value $<0.05$. 
Derivation of GSVA gene sets. All input gene sets used for GSVA analysis can be found in Supplementary Table 3. Cellular and inflammatory modules and IFN-induced gene sets were previously derived ${ }^{15,16}$. Additional inflammatory pathways (NLRP3 Inflammasome, Classical Complement, Alternative Complement, and Lectininduced Complement were curated from the Molecular Signatures Database. Other signatures were derived from various publications $\mathrm{s}^{29,35-38,70}$.

Additional hematopoietic cellular gene signatures (monocyte, myeloid, and neutrophil) were derived from I-Scope, a tool developed to identify immune cell specific genes in big data gene expression analyses. Nonhematopoietic fibroblast and lung cell gene sets were derived from T-Scope, a tool developed to identify genes specific for 45 non-hematopoietic cell types or tissues in big gene expression datasets. The T-Scope database contains 1,234 transcripts derived initially from 10,000 tissue enriched and 8,000 cell line enriched genes listed in the Human Protein Atlas. From the list of 18,000 potential tissue or cell specific genes, housekeeping genes and genes differentially expressed in 40 hematopoietic cell datasets were removed. The final gene lists were checked against available single cell analyses to confirm cellular specificity.

Nine single-cell RNA-seq lung cell populations (AT1, AT2, Ciliated, Club, Endothelial, Fibroblasts, Immuno Monocytes, Immuno T Cells, and Lymphatic Endothelium) were downloaded from the Eils Lung Tissues set ${ }^{71}$ accessed by the UC Santa Cruz Genome Browser (https://eils-lung.cells.ucsc.edu). Genes occurring in more than one cell type were removed. Additionally, genes known to be expressed by immune cells were removed. The Eils Lung Tissues set Immuno Monocyte, Immuno T Cell, Fibroblast, and Lymphatic Endothelium categories were not employed in further analyses.

Apoptosis and NFkB gene signatures were derived and modified from Ingenuity Pathway Analysis pathways Apoptosis Signaling and NFkB Signaling. ROS-protection was derived from Biologically Informed GeneClustering (BIG-C).

Network analysis and visualization. Visualization of protein-protein interaction and relationships between genes within datasets was done using Cytoscape (version 3.6.1) software (Supplementary Table 4). Briefly, STRING (version 1.3.2) generated networks were imported into Cytoscape (version 3.6.1) and partitioned with MCODE via the clusterMaker2 (version 1.2.1) plugin. For PPIs in Fig. 4a-c, STRING settings were adjusted to high confidence (0.7), for PPIs in Fig. 4d-f, settings were relaxed to medium confidence (0.4). All PPIs were generated without the neighborhood or textmining features. For some PPIs, the average interaction strength using STRING-based cumulative interaction scores was used to determine the strength of interaction between clusters.

Functional and cellular enrichment analysis. Functional enrichment of clusters was performed using Biologically Informed Gene-Clustering (BIG-C), which was developed to understand the potential biological meaning of large lists of genes ${ }^{72}$. Genes are clustered into 53 categories based on their most likely biological function and/or cellular localization based on information from multiple on-line tools and databases including UniProtKB/Swiss-Prot, GO terms, KEGG Pathways, MGI database, NCBI PubMed, and the Interactome. Hematopoietic cellular enrichment was performed using I-Scope, a tool developed to identify immune cell specific genes in big data gene expression analyses. Statistically significant enriched types of cell types in DEGs are determined by Fisher's Exact test overlap $p$ value and then determining an Odds Ratio of enrichment.

Derivation of co-expressed myeloid subpopulations in each compartment. Co-expression analyses were conducted in R. Sample (control and patient) log2 expression values for each gene of the 221 identified monocyte/myeloid cell genes in were analyzed for their Pearson correlation coefficient in each tissue compartment (blood, lung, and airway) using the Cor function. Of note, only 196 of 221 genes had changes in gene expression in at least one tissue by RNA-seq. Pearson correlations for these 196 genes were hierarchically clustered by their Euclidian distance into 2 clusters $(\mathrm{k}=2)$ using the heatmap. 2 function in $\mathrm{R}$. This resulted in $2 \mathrm{Mo} /$ myeloid co-expressed clusters in each compartment corresponding to increased and decreased gene sets. The upregulated co-expressed genes were used to define the A1, A2, and A3 myeloid subpopulations from the blood, lung, and airway compartments, respectively (Supplementary Table 5). The co-expressed myeloid populations in each compartment (A1-A3) were then evaluated for enrichment by GSVA.

Inter-compartment myeloid gene comparisons. To compare relative expression of the 196 myeloidspecific genes among compartments, HTS filtered $\log 2$ expression values for each gene were normalized to the average expression of FCGR1A, FCGR2A, and FCGR2C in each sample. Welch's t-test was used to calculate the significant differences in normalized gene expression between cohorts. Effect sizes were computed between cohorts using the cohen.d function with Hedges' correction in R.

Monocle. Trajectory analyses were performed with Monocle ${ }^{32-34}$ version 2.14.0 in R. Gene expression values for 621 genes related to myeloid cell differentiation and function including cell surface and secreted markers, M1 and M2 markers, metabolism, and IFN genes were selected as a curated input list (Supplementary Table 5). The HTS filtered log2 expression values for each of these genes in each sample for each tissue type (PBMCCoV2, Lung-CoV2, and BAL-CoV2) was normalized by the average log2 expression of FCGR1A, FCGR2A, and FCGR2C in that particular sample as described above. Normalized expression of these genes was used as the input expression data for Monocle. The CellDataSet was created with parameters of lowerDetectionLimit $=0.01$ and expressionFamily = uninormal(). Dimensions were reduced using the DDRTree method, and the max_components parameter was set to 2 . Cell state was ordered based upon the state corresponding to PBMC-CoV2. 
Linear regression analysis. Simple linear regression between calculated myeloid subpopulation A1, A2, and A3 GSVA scores and biological functions or signaling pathway GSVA scores was performed in GraphPad Prism Version 8.4.2. In all analyses where pathway genes (e.g. classical complement) were also myeloid cell genes, these genes were removed from the myeloid GSVA score for that comparison and kept in the pathway GSVA score. For each regression analysis, the Goodness of Fit is displayed as the R squared value and the p-value testing the significant of the slope is displayed. All p-values are displayed with 3 digits and rounded-up unless rounding changes significance.

Ingenuity pathway analysis. The canonical pathway and upstream regulator functions of IPA core expression analysis tool (Qiagen) were used to interrogate DEG lists. Canonical pathways and upstream regulators were considered significant if $\mid$ Activation Z-Score $\mid \geq 2$ and overlap p-value $<0.01$. Chemical reagents, chemical toxicants, and endogenous non-mammalian ligands were culled from all upstream regulator analyses.

Drug-target matching. IPA-predicted upstream regulators were annotated with respective targeting drugs and compounds to elucidate potential useful therapies in SARS-CoV2. Drugs targeting gene products of interest by both direct and indirect targeting mechanisms were sourced by Combined Lupus Treatment Scoring (CoLTS)-scored drugs ${ }^{73}$, the Connectivity Map via the drug repurposing tool, DrugBank, and literature mining. Similar methods were employed to determine information about drugs and compounds, including mechanism of action and stage of clinical development. The drug repurposing tool was accessed at clue.io/repurposing-app.

Analysis of COVID-19 PBMC, lung, and BAL DEG profiles via CLUE. DEGs from PBMC-CoV2 vs. PBMC-CTL, Lung-CoV2 vs. Lung-CTL, and BAL-CoV2 vs. PBMC-CoV2 were used as input for the CMaP and LINCS Unified Environment (CLUE) cloud-based connectivity map analysis platform (https://clue.io/conne ctopedia/). Top upregulated and downregulated DEGs from each signature as determined by magnitude of $\log _{2}$ fold change were sequentially entered into CLUE until 150 of each were accepted for analysis to determine drugs, compounds, small molecules, and other perturbagens that mimic or oppose the uploaded COVID-19 gene expression signatures. Resultant drugs and compounds with negative connectivity scores in the $[-75,-100]$ range were analyzed to include results with high confidence of antagonizing COVID-19 gene expression profiles.

\section{Data availability}

The datasets analyzed in this study are available from the China National Center for Bioinformation's National Genomics Data Center, https://bigd.big.ac.cn/gsa/browse/CRA002390, and in the NCBI GEO repository https:// www.ncbi.nlm.nih.gov/bioproject/PRJNA615032. Additional data generated or analyzed during this study are included in this published article (and its Supplementary Information files).

Received: 11 June 2020; Accepted: 3 March 2021

Published online: 29 March 2021

\section{References}

1. Greenberg, S. B. Update on human rhinovirus and coronavirus infections. Semin. Respir. Crit. Care Med. 37, 555-571 (2016).

2. Cui, J., Li, F. \& Shi, Z. L. Origin and evolution of pathogenic coronaviruses. Nat. Rev. Microbiol. 17, 181-192 (2019).

3. Fung, T. S. \& Liu, D. X. Human Coronavirus: Host-Pathogen Interaction. 529-560 (2019).

4. Zhang, B. et al. Clinical characteristics of 82 death cases with COVID-19. medRxiv 2020.02.26.20028191 (2020). https://doi.org/ $10.1101 / 2020.02 .26 .20028191$

5. Chen, G. et al. Clinical and immunologic features in severe and moderate coronavirus disease 2019. J. Clin. Invest. https://doi.org/ 10.1172/JCI137244 (2020).

6. Lazear, H. M., Schoggins, J. W. \& Diamond, M. S. Shared and distinct functions of type I and type III interferons. Immunity 50, 907-923 (2019).

7. Kelley, N., Jeltema, D., Duan, Y. \& He, Y. The NLRP3 inflammasome: An overview of mechanisms of activation and regulation. Int. J. Mol. Sci. 20, 1-24 (2019).

8. Newton, A. H., Cardani, A. \& Braciale, T. J. The host immune response in respiratory virus infection: Balancing virus clearance and immunopathology. Semin. Immunopathol. 38, 471-482 (2016).

9. McGonagle, D., Sharif, K., O’Regan, A. \& Bridgewood, C. The Role of Cytokines including Interleukin-6 in COVID-19 induced Pneumonia and Macrophage Activation Syndrome-Like Disease. Autoimmun. Rev. https://doi.org/10.1016/j.autrev.2020.102537 (2020).

10. Crayne, C. B., Albeituni, S., Nichols, K. E. \& Cron, R. Q. The immunology of macrophage activation syndrome. Front. Immunol. 10, 1-11 (2019).

11. Xiong, Y. et al. Transcriptomic characteristics of bronchoalveolar lavage fluid and peripheral blood mononuclear cells in COVID19 patients. Emerg. Microbes Infect. 9, 761-770 (2020).

12. Blanco-Melo, D. et al. Imbalanced host response to SARS-CoV-2 drives development of COVID-19. Cell https://doi.org/10.1016/j. cell.2020.04.026 (2020).

13. Wei, L. et al. Viral invasion and type I interferon response characterize the immunophenotypes during covid-19 infection. SSRN Electron. J. https://doi.org/10.2139/ssrn.3564998 (2020).

14. Gu, Z., Eils, R. \& Schlesner, M. Complex heatmaps reveal patterns and correlations in multidimensional genomic data. Bioinformatics 32, 2847-2849 (2016).

15. Catalina, M. D., Bachali, P., Geraci, N. S., Grammer, A. C. \& Lipsky, P. E. Gene expression analysis delineates the potential roles of multiple interferons in systemic lupus erythematosus. Commun. Biol. 2, 140 (2019).

16. Catalina, M. D. et al. Patient ancestry significantly contributes to molecular heterogeneity of systemic lupus erythematosus. JCI Insight $\mathbf{5}$ (2020).

17. Wen, W. et al. Immune cell profiling of COVID-19 patients in the recovery stage by Single-cell sequencing. Cell Discov. 6(1) (2020).

18. Kegerreis, B. J. et al. Genomic identification of low-density granulocytes and analysis of their role in the pathogenesis of systemic lupus erythematosus. J. Immunol. 202, 3309-3317 (2019). 
19. Schulte-Schrepping, J. et al. Severe COVID-19 is marked by a dysregulated myeloid cell compartment. Cell 182, 1419-1440.e23 (2020).

20. Aschenbrenner, A. C. et al. Disease severity-specific neutrophil signatures in blood transcriptomes stratify COVID-19 patients. medRxiv 2020.07.07.20148395 (2020). https://doi.org/10.1101/2020.07.07.20148395

21. Trouillet-Assant, S. et al. Type I IFN immunoprofiling in COVID-19 patients. J. Allergy Clin. Immunol. https://doi.org/10.1016/j. jaci.2020.04.029 (2020).

22. Fischer, H., Tschachler, E. \& Eckhart, L. Pangolins lack IFIH1/MDA5, a cytoplasmic RNA sensor that initiates innate immune defense upon coronavirus infection. Front. Immunol. 11, 939 (2020).

23. Bojkova, D. et al. Proteomics of SARS-CoV-2-infected host cells reveals therapy targets. Nature https://doi.org/10.1038/s41586020-2332-7 (2020).

24. Vazquez, C. \& Horner, S. M. MAVS Coordination of Antiviral Innate Immunity. J. Virol. 89, 6974-6977 (2015).

25. Lindell, D. M., Lane, T. E. \& Lukacs, N. W. CXCL10/CXCR3-mediated responses promote immunity to respiratory syncytial virus infection by augmenting dendritic cell and CD8(+) T cell efficacy. Eur. J. Immunol. 38, 2168-2179 (2008).

26. Teijaro, J. R. The role of cytokine responses during influenza virus pathogenesis and potential therapeutic options. Curr. Top. Microbiol. Immunol. 386, 3-22 (2015).

27. Sungnak, W. et al. SARS-CoV-2 entry factors are highly expressed in nasal epithelial cells together with innate immune genes. Nat. Med. https://doi.org/10.1038/s41591-020-0868-6 (2020).

28. Morris, J. H. et al. clusterMaker: A multi-algorithm clustering plugin for cytoscape. BMC Bioinform. 12, 436 (2011).

29. Liao, M. et al. The landscape of lung bronchoalveolar immune cells in COVID-19 revealed by single-cell RNA sequencing. medRxiv 2020.02.23.20026690 (2020). https://doi.org/10.1101/2020.02.23.20026690

30. Suzuki, T. et al. Pulmonary macrophage transplantation therapy. Nature 514, 450-454 (2014).

31. Joshi, P. C. et al. GM-CSF receptor expression and signaling is decreased in lungs of ethanol-fed rats. Am. J. Physiol. Lung Cell. Mol. Physiol. 291, L1150-L1158 (2006).

32. Trapnell, C. et al. The dynamics and regulators of cell fate decisions are revealed by pseudotemporal ordering of single cells. Nat. Biotechnol. 32, 381-386 (2014).

33. Qiu, X. et al. Single-cell mRNA quantification and differential analysis with Census. Nat. Methods 14, 309-315 (2017).

34. Qiu, X. et al. Reversed graph embedding resolves complex single-cell trajectories. Nat. Methods 14, 979-982 (2017).

35. Reyfman, P. A. et al. Single-cell transcriptomic analysis of human lung provides insights into the pathobiology of pulmonary fibrosis. Am. J. Respir. Crit. Care Med. 199, 1517-1536 (2019).

36. Hubbard, E. L. et al. Analysis of gene expression from systemic lupus erythematosus synovium reveals a profile of activated immune cells and inflammatory pathways. bioRxiv 2020.06.19.123307 (2020). https://doi.org/10.1101/2020.06.19.123307

37. Kuo, D. et al. HBEGF+ macrophages in rheumatoid arthritis induce fibroblast invasiveness. Sci. Transl. Med. 11, eaau8587 (2019).

38. Martinez, F. O., Gordon, S., Locati, M. \& Mantovani, A. Transcriptional profiling of the human monocyte-to-macrophage differentiation and polarization: New molecules and patterns of gene expression. J. Immunol. 177, 7303-7311 (2006).

39. Tay, M. Z., Poh, C. M., Rénia, L., MacAry, P. A. \& Ng, L. F. P. The trinity of COVID-19: Immunity, inflammation and intervention. Nat. Rev. Immunol. https://doi.org/10.1038/s41577-020-0311-8 (2020).

40. Corley, M. J., Sugai, C., Schotsaert, M., Schwartz, R. E. \& Ndhlovu, L. C. Comparative \&lt;em\&gt;in vitro\&lt;/em\&gt; transcriptomic analyses of COVID-19 candidate therapy hydroxychloroquine suggest limited immunomodulatory evidence of SARS-CoV-2 host response genes. bioRxiv 2020.04.13.039263 (2020). https://doi.org/10.1101/2020.04.13.039263

41. Viola, A., Munari, F., Sánchez-Rodríguez, R., Scolaro, T. \& Castegna, A. The metabolic signature of macrophage responses. Front. Immunol. 10, 1-16 (2019).

42. Huang, C. et al. Clinical features of patients infected with 2019 novel coronavirus in Wuhan, China. Lancet 395, 497-506 (2020).

43. Barnes, B. J. et al. Targeting potential drivers of COVID-19: Neutrophil extracellular traps. J. Exp. Med. 217, 1-7 (2020).

44. Thierry, A. R. \& Roch, B. NETs by-products and extracellular DNA may play a key role in COVID-19 pathogenesis: Incidence on patient monitoring and therapy (2020). https://doi.org/10.20944/preprints202004.0238.v1

45. Carmona-Rivera, C. \& Kaplan, M. J. Low-density granulocytes: A distinct class of neutrophils in systemic autoimmunity. Semin. Immunopathol. 35, 455-463 (2013).

46. Tipping, P. G., Campbell, D. A., Boyce, N. W. \& Holdsworth, S. R. Alveolar macrophage procoagulant activity is increased in acute hyperoxic lung injury. Am. J. Pathol. 131, 206-212 (1988).

47. Wichmann, D. et al. Autopsy findings and venous thromboembolism in patients with COVID-19: A prospective cohort study. Ann. Intern. Med. https://doi.org/10.7326/M20-2003 (2020).

48. Allard, B., Panariti, A. \& Martin, J. G. Alveolar macrophages in the resolution of inflammation, tissue repair, and tolerance to infection. Front. Immunol. 9, 1777 (2018).

49. Chen, N. et al. Epidemiological and clinical characteristics of 99 cases of 2019 novel coronavirus pneumonia in Wuhan, China: A descriptive study. Lancet 395, 507-513 (2020).

50. Qin, C. et al. Dysregulation of immune response in patients with COVID-19 in Wuhan, China. Clin. Infect. Dis. 2019, 4-10 (2020).

51. Xu, Z. et al. Pathological findings of COVID-19 associated with acute respiratory distress syndrome. Lancet Respir. Med. 8, 420-422 (2020).

52. He, Z. et al. Effects of severe acute respiratory syndrome (SARS) coronavirus infection on peripheral blood lymphocytes and their subsets. Int. J. Infect. Dis. 9, 323-330 (2005).

53. Wu, F. et al. Neutralizing antibody responses to SARS-CoV-2 in a COVID-19 recovered patient cohort and their implications. medRxiv 2020.03.30.20047365 (2020). https://doi.org/10.1101/2020.03.30.20047365

54. Iwasaki, A. \& Yang, Y. The potential danger of suboptimal antibody responses in COVID-19. Nat. Rev. Immunol. https://doi.org/ 10.1038/s41577-020-0321-6 (2020).

55. Liu, L. et al. Anti-spike IgG causes severe acute lung injury by skewing macrophage responses during acute SARS-CoV infection. JCI Insight 4, 1-19 (2019).

56. Heron, M. et al. Bronchoalveolar lavage cell pattern from healthy human lung. Clin. Exp. Immunol. 167, 523-531 (2012).

57. Hamacher, J. et al. Tumor necrosis factor- $a$ and angiostatin are mediators of endothelial cytotoxicity in bronchoalveolar lavages of patients with acute respiratory distress syndrome. Am. J. Respir. Crit. Care Med. 166, 651-656 (2002).

58. Pairo-Castineira, E. et al. Genetic mechanisms of critical illness in Covid-19. Nature https://doi.org/10.1038/s41586-020-03065-y (2020).

59. Sun, X. et al. Cytokine storm intervention in the early stages of COVID-19 pneumonia. Cytokine Growth Factor Rev. https://doi. org/10.1016/j.cytogfr.2020.04.002 (2020).

60. Pedersen, S. F. \& Ho, Y.-C. SARS-CoV-2: A storm is raging. J. Clin. Invest. 130, 2202-2205 (2020).

61. Voiriot, G. et al. Interleukin-6 displays lung anti-inflammatory properties and exerts protective hemodynamic effects in a doublehit murine acute lung injury. Respir. Res. 18, 64 (2017).

62. Mehta, P. et al. COVID-19: Consider cytokine storm syndromes and immunosuppression. Lancet (London, England) 395, 1033$1034(2020)$.

63. Gao, T. et al. Highly pathogenic coronavirus N protein aggravates lung injury by MASP-2-mediated complement over-activation. medRxiv 2020.03.29.20041962 (2020). https://doi.org/10.1101/2020.03.29.20041962 
64. Patterson, B. K. et al. Disruption of the CCL5/RANTES-CCR5 pathway restores immune homeostasis and reduces plasma viral load in critical COVID-19. medRxiv 2020.05.02.20084673 (2020). https://doi.org/10.1101/2020.05.02.20084673

65. Middeldorp, S. et al. Incidence of venous thromboembolism in hospitalized patients with COVID-19. J. Thromb. Haemost. https:// doi.org/10.1111/jth.14888 (2020).

66. Klok, F. A. et al. Incidence of thrombotic complications in critically ill ICU patients with COVID-19. Thromb. Res. https://doi.org/ 10.1016/j.thromres.2020.04.013 (2020)

67. Geleris, J. et al. Observational Study of Hydroxychloroquine in Hospitalized Patients with Covid-19. N. Engl. J. Med. (2020).

68. Anders, S. \& Huber, W. Differential expression analysis for sequence count data. Genome Biol. 11, R106 (2010).

69. Hänzelmann, S., Castelo, R. \& Guinney, J. GSVA: Gene set variation analysis for microarray and RNA-Seq data. BMC Bioinform. 14, 7 (2013).

70. Kotliarov, Y. et al. Broad immune activation underlies shared set point signatures for vaccine responsiveness in healthy individuals and disease activity in patients with lupus. Nat. Med. https://doi.org/10.1038/s41591-020-0769-8 (2020).

71. Lukassen, S. et al. SARS-CoV-2 receptor ACE2 and TMPRSS2 are predominantly expressed in a transient secretory cell type in subsegmental bronchial branches. bioRxiv 2020.03.13.991455 (2020). https://doi.org/10.1101/2020.03.13.991455

72. Labonte, A. C. et al. Identification of alterations in macrophage activation associated with disease activity in systemic lupus erythematosus. PLoS ONE 13, e0208132 (2018).

73. Grammer, A. C. et al. Drug repositioning in SLE: Crowd-sourcing, literature-mining and big data analysis. Lupus 25, 1150-1170 (2016).

\section{Acknowledgements}

We thank the patients who provided samples for this study as well as the authors of previous studies ${ }^{11,12}$ who made their data publicly available and facilitated our analyses.

\section{Author contributions}

Conceptualization, P.E.L., A.C.G.; Methodology, A.R.D. and P.E.L.; Software, P.B., K.A.O, and K.M.K.; Formal Analysis, A.R.D., P.B., K.A.O., K.M.K., and E.L.H.; Data Curation, P.B.; Writing-Original Draft, A.R.D., K.A.O., K.M.K., and E.L.H.; Writing-Review \& Editing, A.R.D., K.A.O., K.M.K., E.L.H., A.C.L., R.R., S.S., A.C.G., and P.E.L.; Visualization, A.R.D., P.B., K.A.O., K.M.K., E.L.H., and A.C.L.; Supervision, A.R.D. and P.E.L.; Project Administration, P.E.L.; Funding Acquisition, A.C.G.

\section{Competing interests}

The authors declare no competing interests.

\section{Additional information}

Supplementary Information The online version contains supplementary material available at https:/doi.org/ 10.1038/s41598-021-86002-X.

Correspondence and requests for materials should be addressed to A.R.D. or P.E.L.

Reprints and permissions information is available at www.nature.com/reprints.

Publisher's note Springer Nature remains neutral with regard to jurisdictional claims in published maps and institutional affiliations.

(i) Open Access This article is licensed under a Creative Commons Attribution 4.0 International License, which permits use, sharing, adaptation, distribution and reproduction in any medium or format, as long as you give appropriate credit to the original author(s) and the source, provide a link to the Creative Commons licence, and indicate if changes were made. The images or other third party material in this article are included in the article's Creative Commons licence, unless indicated otherwise in a credit line to the material. If material is not included in the article's Creative Commons licence and your intended use is not permitted by statutory regulation or exceeds the permitted use, you will need to obtain permission directly from the copyright holder. To view a copy of this licence, visit http://creativecommons.org/licenses/by/4.0/.

(c) The Author(s) 2021 